\title{
On the arithmetic of D-brane superpotentials. Lines and conics on the mirror quintic
}

\author{
JOHANNES WALCHER
}

\begin{abstract}
Irrational invariants from D-brane superpotentials are pursued on the mirror quintic, systematically according to the degree of a representative curve. Lines are completely understood: the contribution from isolated lines vanishes. All other lines can be deformed holomorphically to the van Geemen lines, whose superpotential is determined via the associated inhomogeneous Picard-Fuchs equation. Substantial progress is made for conics: the families found by Mustaţă contain conics reducible to isolated lines, hence they have a vanishing superpotential. The search for all conics invariant under a residual $\mathbb{Z}_{2}$ symmetry reduces to an algebraic problem at the limit of our computational capabilities. The main results are of arithmetic flavor: the extension of the moduli space by the algebraic cycle splits in the large complex structure limit into groups each governed by an algebraic number field. The expansion coefficients of the superpotential around large volume remain irrational. The integrality of those coefficients is revealed by a new, arithmetic twist of the di-logarithm: the D-logarithm. There are several options for attempting to explain how these invariants could arise from the A-model perspective. A successful spacetime interpretation will require spaces of BPS states to carry number theoretic structures, such as an action of the Galois group.
\end{abstract}

1. Introduction

2. Lines on the mirror quintic

2.1. $2875=375+2500$

2.2. Inhomogeneous Picard-Fuchs equation

3. Conics on the mirror quintic 
3.2. $S^{3}$-invariant conics

3.3. $\mathbb{Z}_{2} \times \mathbb{Z}_{2}$-invariant conics

292

3.4. Taking advantage of phase symmetries

3.5. $\mathbb{Z}_{2}^{-}$-invariant conics

3.6. Relationships

298

3.6.1 $\mathbb{Z}_{2} \times \mathbb{Z}_{2}$ meets $S^{3}$.

3.6.2 Reducible conics.

300

3.6.3 $\mathbb{Z}_{2}^{-}$meets $\mathbb{Z}_{2}^{+}$.

3.6.4 A family of reducible conics.

3.6.5 Another coincidence.

4. Summary so far

301

5. Expansion in large complex structure limit

5.1. Newton-Puiseux expansion 302

5.2. The first component

5.3. The main component

6. Expansion in large volume limit

6.1. Van Geemen lines

6.2. The D-logarithm

6.3. First component, first group

6.4. D-logarithm $\bmod k^{2}$

6.5. First component, second group 


\section{Introduction}

The purpose of this paper is to continue pushing the limit of the calculation of D-brane superpotentials using the methods developed in $[1,2]$. The object of study is the value

$$
\mathcal{W}(z)=\left.\mathcal{W}(u ; z)\right|_{\partial_{u} \mathcal{W}=0}
$$

of the spacetime superpotential, at the critical point in the open string direction, compactly denoted by $u$, as a function of the closed string moduli, $z$. Note right away that (1.1) does not depend on the ambiguous off-shell parameterization of the open string moduli space, and is as such a true holomorphic invariant of the underlying D-brane configuration. ${ }^{1}$

As in $[2,3]$, we have in mind a comparison between three different points of view on $\mathcal{W}(z)$. The mathematically best defined framework is the B-model. In that context, $z \in M$ is the complex structure parameter of a family of Calabi-Yau three-folds $\mathcal{Y} \rightarrow M$. We denote the manifold of modulus $z$ by $Y_{z}$, or simply $Y$ when $z$ is generic. In standard cases, the D-brane is associated with a family of holomorphic vector bundles $\mathcal{E}$ over $\mathcal{Y}$, or more generally an object in the derived category $D^{b}(Y)$ varying appropriately with $z$. The off-shell superpotential $\mathcal{W}(u ; z)$ which measures the obstructions to deforming $\mathcal{E}$ in the open string directions, $u$, as a function of the closed string moduli, $z$, is given by the holomorphic Chern-Simons functional, or an appropriate extension or dimensional reduction thereof for more general objects of $D^{b}(Y)$. A general effective description of the on-shell superpotential involves the (truncated) normal function $\nu_{\mathcal{C}}(z)$ associated to a family of algebraic cycles $\mathcal{C}$ that if required can be obtained as the algebraic second Chern class of $\mathcal{E}$. See [1] for detailed explanations.

\footnotetext{
${ }^{1}$ For a perhaps not over-simplified way to see arithmetic arising in this context, imagine that $\mathcal{W}(u)$ is a polynomial with integer coefficients, and the superpotential of a supersymmetric theory with four supercharges. Then the supersymmetric vacua and the critical values $\left.\mathcal{W}\right|_{\partial \mathcal{W}(u)=0}$, which are the actual holomorphic invariants encoded in $\mathcal{W}(u)$, generically belong to a finite algebraic extension of the rationals. The statement of arithmeticy is somewhat different in the context of attractors in supergravity [4], where one looks at critical points of transcendental functions (periods).
} 
Near a singular point of maximal unipotent monodromy of the family $\mathcal{Y}$, one can obtain a dual, A-model, point of view on $\mathcal{W}$. The D-brane there is manufactured starting from a Lagrangian submanifold, $L$, of the mirror Calabi-Yau $X$, together with a flat connection. While classically the deformations of $L$ are unobstructed, worldsheet instanton corrections may induce a superpotential that lifts the D-brane's moduli space. The critical points of the superpotential can be identified with unobstructed objects of the Fukaya category. The difficult problem is to properly count the holomorphic discs with boundary on the Lagrangian that give rise to that superpotential.

The third point of view, developed by Ooguri and Vafa [5], comes from embedding into the type IIA/B superstring compactified on $X / Y$ for A/B-model respectively. One considers the effective two-dimensional theory living on a D-brane partially wrapped on $L$ or $\mathcal{E}$, and extended along a two-dimensional subspace $\mathbb{R}^{2} \subset \mathbb{R}^{4}$. According to Ooguri and Vafa [5], the superpotential $\mathcal{W}$ not only controls the supersymmetric vacua, but is also related, via its expansion in the appropriate limits, to the BPS content of the two-dimensional theory. Mirror symmetry relates the choice of $(X, L)$ and $(\mathcal{Y}, \mathcal{E})$ and implies that the superpotentials computed in A- and B-model are identical. Duality with M-theory explains the relation to the BogomolonyiPrasad-Sommerfield (BPS) content of the two-dimensional theory.

The essence of the mirror correspondence is that while the calculations from A-model or spacetime perspective are forbiddingly difficult in general, the B-model is relatively straightforward. On the other hand, the interpretation in terms of novel geometric invariants is best (although by no means completely) understood in the A-model, and most interesting, from the spacetime perspective. Interesting mathematics is everywhere. In this paper, we will present new B-model calculations whose successful A-model and spacetime interpretation could force a significant extension of the reach of these models, into number theory.

Detailed calculations in open string mirror symmetry are now available in a variety of situations. Motivated and guided by a number of works involving non-compact manifolds [6-9], a quantitative mirror correspondence involving D-branes on the quintic was established in [3]. Further works involving compact manifolds include [10-18]. In all these examples, the underlying manifold was selected from the beginning of the long and wellknown list of complete intersections in toric varieties, for instance hypersurfaces in weighted projective spaces. (The noteworthy exception is [17], which deals with Pfaffian Calabi-Yau manifolds.) The choice of cycle in the B-model followed by exploiting divisibility properties of weights of specific monomials. In a sense, these D-branes were as close as they could be, to the 
"toric branes" that are customarily studied in the context of non-compact examples. Somewhat by accident, a subset of those cycles turned out to be relevant as the mirror of real slices of the A-model manifold.

In this work, we return to the quintic Calabi-Yau, with a somewhat different rationale for selecting the D-branes. As far as A-model is concerned, methods for constructing Lagrangian submanifolds of compact Calabi-Yau manifolds are in short supply. As far as the B-model, holomorphic vector bundles are much easier to produce, perhaps surpassed in simplicity only by matrix factorizations. The simplest constructions, however, pull vector bundles back from projective space, which results in rather boring superpotentials (at least on-shell). Matrix factorizations have the additional disadvantage that they do not come with a readily usable version of holomorphic Chern-Simons. To obtain an interesting holomorphic invariant, what we really need is a non-trivial algebraic cycle class. So we might as well construct the representative cycle $\mathcal{C}$, directly, and then calculate as in [1]. Although this seems like a reasonable approach to finding new D-branes, it is not systematically developed. So a large initial portion of this work is concerned with identifying appropriate $\mathcal{C}$.

There are several motivations for pursuing this direction. First of all, these methods will definitely take us further away from the set of toric branes, and we can prepare ourselves for unexpected new phenomena. (As mentioned above, the calculations in [17] are outside the toric realm. However, the complication there is introduced in the bulk, i.e., at the level of the Calabi-Yau, while the D-branes follow the simpler pattern, conjecturally related to the real A-branes.) In due course, these results will shape expectations in investigating A-branes and their invariants.

Another broad motivation for this work is a more systematic exploration of the set of all possible D-branes for fixed closed string background, from the holomorphic point of view. This is related on the one hand to speculations about background independence in this context [19]. On the other hand, a better overview over the set of all D-branes might also be important for realizing open/closed string correspondence on compact manifolds. In the context of the topological string, the invariant holomorphic information contained in the superpotential could be the minimal amount necessary.

To organize the advance, we recall again that on general grounds, all possible on-shell superpotentials in the sense of (1.1) are realized geometrically as truncated normal functions. On families of Calabi-Yau three-folds, we are looking for the image of the Chow group $\mathrm{CH}^{2}(Y)$ of algebraic cycles modulo rational equivalence, under the Abel-Jacobi map. A natural filtration on $\mathrm{CH}^{2}(Y)$ is the minimal degree of a curve representing a given cycle 
class. This minimal degree is our organizing principle. In this paper, we will proceed up to degrees 1 and 2 on the mirror quintic, which as it turns out are already immensely interesting.

We begin in Section 2 with a review of what is known about lines on the mirror quintic, and then proceed to calculate the inhomogeneous PicardFuchs equation associated with van Geemen lines. The dissection of conics in Section 3 is perhaps hard to follow, so we have attempted a shorter summary in Section 4. The first part of Section 5 is warmly recommended, as well as a glance at Equation (5.27). Section 6 contains the main results, and Section 7 is a best attempt at an interpretation.

\section{Lines on the mirror quintic}

Investigations into low degree curves on the quintic have a long history reaching back before the beginning of mirror symmetry, and provided important information regarding the latter's enumerative predictions. Instead of the generic quintic, we are here concerned with curves on the special oneparameter family of mirror quintics. This family is related to the vanishing locus of the polynomial

$$
W=\frac{x_{1}^{5}}{5}+\frac{x_{2}^{5}}{5}+\frac{x_{3}^{5}}{5}+\frac{x_{4}^{5}}{5}+\frac{x_{5}^{5}}{5}-\psi x_{1} x_{2} x_{3} x_{4} x_{5}
$$

in five homogeneous complex coordinates $\left(x_{1}, \ldots, x_{5}\right)$, and the one parameter, $\psi$. We denote by $Y$ the generic quintic in $\mathbb{P}^{4}$. By $Y_{\psi}$ we denote the member of the Dwork family $\mathcal{Y} \rightarrow M$ for fixed $\psi$, given by $\{W=0\} \subset \mathbb{P}^{4}$. The actual mirror quintic is of course the resolution of the quotient of $Y_{\psi}$ by $\left(\mathbb{Z}_{5}\right)^{3}$. This is useful to keep in mind, but will play only a minor role in the present discussion.

\section{1. $2875=375+2500$}

The space of lines on the one-parameter family of mirror quintics has been investigated thoroughly by Mustaţă [20], building on the earlier work [21]. The main results of [20] is the following: for fixed generic $\psi$, the quintic $Y_{\psi}$ contains precisely 375 isolated lines, and 2 (isomorphic) families of lines, each parameterized by a genus 626 curve. One of the isolated lines is the coordinate line

$$
x_{1}+x_{2}=0, \quad x_{3}+x_{4}=0, \quad x_{5}=0,
$$


while the others are obtained by either permuting the $\left(x_{1}, \ldots, x_{5}\right)$, or inserting a fifth root of unity in the first two equations. This leads to the count $5 ! / 2^{3} \times 5^{2}=375$.

Special members of the families can easily be written down. If $\omega$ is a non-trivial third root of unity, and $(a, b)$ satisfy the equations

$$
a^{5}+b^{5}=27, \quad \psi a b=6,
$$

then the line

$$
\begin{aligned}
x_{1}+\omega x_{2}+\omega^{2} x_{3} & =0, \\
a\left(x_{1}+x_{2}+x_{3}\right)-3 x_{4} & =0, \\
b\left(x_{1}+x_{2}+x_{3}\right)-3 x_{5} & =0,
\end{aligned}
$$

lies on the quintic $Y_{\psi}$. (This is easiest to see by parameterizing solutions of (2.4) as

$$
\left(x_{1}, x_{2}, x_{3}, x_{4}, x_{5}\right)=\left(u+v, u+\omega v, u+\omega^{2} v, a u, b u\right),
$$

where $(u, v)$ are homogeneous coordinates on $\mathbb{P}^{1}$. Then plugging this into (2.1), and using $1+\omega+\omega^{2}=0$, gives $u^{5}\left(3+a^{5}+b^{5}-5 \psi a b\right)+u^{2} v^{3}(30-$ $5 \psi a b)=0$ which directly yields (2.3).)

Taking into account the phase and permutation symmetries, one obtains a set of 5000 lines, called van Geemen lines. This being more that the number of lines on the generic quintic three-fold, which is 2875 , was historically important because it allowed the conclusion that there exist families of lines on the generic member $Y_{\psi}$ of the family (2.1). The structure of the families at fixed $\psi$, as mentioned above, was worked out only more recently, and consists of two curves of genus 626 .

Anticipating results of our Abel-Jacobi calculations in the next subsection, we note how it will distinguish the two families of lines: exchanging $a$ and $b$ is equivalent to exchanging $x_{4}$ and $x_{5}$, from which the holomorphic three-form and hence the normal function, and superpotential, pick up a minus sign. In a slightly different way, changing the choice of third root of unity, i.e., the transformation

$$
\omega \mapsto \omega^{2}
$$

is equivalent to exchanging $x_{2}$ and $x_{3}$, and hence also inverts the Abel-Jacobi image. 
The global structure of the families of lines, with varying $\psi$, was also worked out in [20]: the curves parameterizing the families containing the van Geemen lines fit together to a single smooth irreducible surface, whose Stein factorization (i.e., the collapse of the connected components in the fibers) gives a double cover of $\psi$-space, with branch points at $\psi=0$, and $\psi^{5}=\frac{128}{3}$. This is the discriminant locus of the Equations (2.3). In particular, the two families that are distinguished for fixed $\psi$ are exchanged as one moves around in the complex structure moduli space. Quite importantly however, $\psi=\infty$ is not a branch point, so in particular, the choice of third root of unity $\omega$ is a good invariant to distinguish classes of D-branes, in the large complex structure limit.

Let us record this as the first instance of an intriguing observation: the mapping (2.6) is nothing but the Galois group of the number field generated by $\omega$ (which is the imaginary quadratic number field $\mathbb{Q}(\sqrt{-3})$ ). The statement about Abel-Jacobi means that the space-time superpotential furnishes a non-trivial representation of the Galois group of the number field over which the D-brane is defined. Following our sober discussion, this might not seem so very surprising. But it has some astonishing consequences that we will explore later on.

To close, we repeat here the count of lines which shows that the isolated lines and the two families account for all rational curves of degree 1 on the family of mirror quintics. (That is, for generic values of $\psi$. At $\psi=0$, for instance, all lines belong to families, as explained in [21], and exploited frequently.) The families contributing with the Euler characteristic of their parameter space gives,

$$
2 \times(2 \times 626-2)+375=2500+375=2875
$$

\subsection{Inhomogeneous Picard-Fuchs equation}

We need to recall a minimum of material from $[1,2]$ : if $Y$ is a Calabi-Yau three-fold, and $C \subset Y$ a holomorphic curve, it makes a contribution to the superpotential [22]

$$
\mathcal{W}(z)=\int^{C} \Omega
$$

This depends on the complex structure parameter $z$ via the choice of a holomorphic three-form $\Omega$ on $Y$, which is to be integrated over a three-chain $\Gamma$ ending on $C$. We have written (2.8) with the understanding that the actual physical invariant quantities are the tensions of BPS domain walls (or masses 
of BPS solitons), which are given by the difference of superpotential values at the critical points, so $\Gamma$ is then the three-chain interpolating between two homologous holomorphic curves.

The reason that (2.8) makes sense even when $C$ is non-trivial in homology is that we calculate $\mathcal{W}$ as a solution of the inhomogeneous Picard-Fuchs equation,

$$
\mathcal{L W}(z)=f(z),
$$

where $\mathcal{L}$ is the Picard-Fuchs differential operator of the family $(\mathcal{Y}, \Omega)$. Since $\mathcal{L} \Omega=d \beta$ is an exact form, the inhomogeneity $f(z)$ originates from integrating $\int_{C} \beta$, together with some contribution from differentiating $C$. Both are clearly local and meaningfully associated to $C$, whether homologically trivial or not.

With respect to the standard choice of $\Omega$, the Picard-Fuchs operator of the quintic mirror has the form

$$
\mathcal{L}=\theta^{4}-5 z(5 \theta+1)(5 \theta+2)(5 \theta+3)(5 \theta+4),
$$

(where $z=(5 \psi)^{-5}$ and $\theta=\frac{d}{d \ln z}$.) The inhomogeneity $f(z)$ was calculated in [1] for the Deligne conics $C_{ \pm}$given by $x_{5}^{2}= \pm \sqrt{5 \psi} x_{1} x_{3}$, within the plane $P=\left\{x_{1}+x_{2}=0, x_{3}+x_{4}=0\right\}$, with the result

$$
f_{ \pm}(z)= \pm \frac{15}{32 \pi^{2}} \sqrt{z}
$$

Since the line (2.2) is residual to those conics in the intersection of $P$ with $Y$, and since, on general grounds, the inhomogeneity associated to $P \cap Y$ vanishes, we can conclude immediately that $f(z)=0$ for any of the 375 isolated lines.

Another general Hodge theoretic result is that curves that can be holomorphically deformed into each other give rise to the same normal function. Mathematically, this is the statement that "algebraic equivalence implies Abel-Jacobi equivalence" (a statement valid for curves on Calabi-Yau threefolds). Physically, finite holomorphic deformations correspond to open string moduli, which are flat directions of the superpotential.

Given this, we conclude that the two families of lines containing the van Geemen lines map under Abel-Jacobi each to a single point in the intermediate Jacobian. It is therefore sufficient to calculate just for the van Geemen lines. Also, as anticipated above, the images of the two families differ just by a sign. 
To calculate $f(z)$ for the van Geemen lines, we may proceed as in $[1,2]$. The key feature to exploit is that any of the lines is part of the intersection of $Y$ with a plane, and that the calculation of $\int_{C} \beta$ localizes to the intersection points of $C$ with the residual quartic in that plane. The difference to $[1,2]$ is that here there is actually a two-parameter family of planes containing any given $C$, so we can make any choice that seems convenient.

The details are straightforwardly executed, and we obtain the inhomogeneity associated with the van Geemen lines,

$$
f_{\text {van Geemen }}(z)=\frac{1+2 \omega}{4 \pi^{2}} \times \frac{32}{45} \times \frac{\frac{63}{\psi^{5}}+\frac{1824}{\psi^{10}}-\frac{512}{\psi^{15}}}{\left(1-\frac{128}{3 \psi^{5}}\right)^{5 / 2}}
$$

$\left(z=(5 \psi)^{-5}\right)$. Notice that as $\omega$ is a non-trivial third root of unity, the inhomogeneity has an overall factor $\sqrt{-3}$ multiplying a function with a power series expansion around $\psi=\infty$ with rational coefficients. The main theme of this paper is to investigate irrationalities in the expansion of the solutions of inhomogeneous Picard-Fuchs equations. As an overall factor, the irrationality might seem rather mild in the present case. This is however dictated by the anticipated sign change under the Galois action $\sqrt{-3} \mapsto-\sqrt{-3}$. Later examples will be more complicated, and also the solutions of the inhomogeneous Picard-Fuchs equation associated to (2.12) will already be quite illuminating, see Section 6.

\section{Conics on the mirror quintic}

The basic framework to search for conics on the quintic is easy to describe, following Katz [23]: the moduli space of conics in $\mathbb{P}^{4}$ is fibered over the Grassmannian $G(3,5)$ of projective planes in $\mathbb{P}^{4}$. The fiber over a plane $A \cong \mathbb{P}^{2} \subset \mathbb{P}^{4}$ is given by the conics in a fixed $\mathbb{P}^{2}$, spanned by the monomials of degree 2 in three homogeneous coordinates on $\mathbb{P}^{2}$, and isomorphic to a copy of $\mathbb{P}^{5}$. We denote a conic in a fixed $\mathbb{P}^{2}$ by $B$. The conic $B \subset A \subset \mathbb{P}^{4}$ is contained in the quintic $Y$ precisely if

$$
Y \cap A=B \cup C
$$

where $C$ is a cubic curve in $A$. 


\subsection{Overview}

In practice, Equation (3.1) means the following: the plane $A$ is defined as the vanishing locus of two linearly independent linear equations in the five homogeneous coordinates, $x_{1}, \ldots, x_{5}$ of $\mathbb{P}^{4}$. Up to taking linear combinations of those two equations, there are six independent parameters entering these equations, which are just (local) coordinates on $G(3,5)$. The equations for $A$ being linear, and non-degenerate, they can be solved for two of the five $x_{i}$ 's, say $x_{4}$ and $x_{5}$. The result can be substituted in the quintic polynomial defining $Y$, yielding a quintic polynomial, $p_{5}$, in three variables. Note that there are 21 different quintic monomials in three variables.

The conic $B \subset A$ is given as the vanishing locus of a quadratic polynomial $p_{2}$ in three variables, say $\left(x_{1}, x_{2}, x_{3}\right)$, and depends on 6 homogeneous parameters. Likewise, the residual cubic is given by a cubic polynomial $p_{3}$, and depends on 10 parameters. Equation (3.1) then is the vanishing of the coefficients of the 21 independent quintic monomials in

$$
p_{5}-p_{2} \cdot p_{3}
$$

Since we may fix the scale of either $p_{2}$ or $p_{3}$ arbitrarily, there are $6+6+10-$ $1=21$ independent parameters entering those 21 equations. (Note that the 10 parameters for $C$ enter linearly in (3.2), which may therefore a priori be reduced to a system of 11 equations in 11 variables. This is the more standard dimensionality of the counting problem.) Generically then, we expect a finite number of solutions. This is in fact true, and there are 609250 conics on the generic quintic [23].

For special quintics, for example a member $Y_{\psi}$ of the one-parameter family of quintics (2.1), there will be some number of isolated solutions, and some number of continuous families. There can also be conics that are reducible to two intersecting lines. When counted appropriately, all these will add up to 609 250. In this paper, our main focus is not on counting solutions, but on performing calculations for particular conics that deform with $Y_{\psi}$ as $\psi$ is varied. Conics that are isolated for fixed $\psi$ will deform to one-parameter families, while families that exist at fixed $\psi$ can either deform as families or be lifted to isolated solutions. Globally these local branches of solutions will fit together to various components of the "relative Hilbert scheme" of conics $\mathcal{H}_{\text {conics }} \rightarrow M$ on the one-parameter family of quintics (2.1), $\mathcal{Y} \rightarrow M$.

The goal in this section is to identify an interesting subset of components of $\mathcal{H}_{\text {conics. }}$. In the next section, we will study the branch structure around the large complex structure limit, $\psi \rightarrow \infty$. To simplify our life, we 
will neglect obstructed families of conics, avoid the singular loci, and all other phenomena that occur at special values of $\psi$.

A fair number of solutions of (3.1) can be found by exploiting the symmetries of the problem. The full symmetry group $G$ of (2.1) consists of the phase symmetries multiplying the $x_{i}$ 's and $\psi$ by fifth roots of unity, and the symmetric group $S^{5}$ that acts by permuting the $x_{i}{ }^{\prime}$ s,

$$
\left(\mathbb{Z}_{5}\right)^{4} \rightarrow G \rightarrow S^{5}
$$

The two subgroups play a somewhat different role in the problem. To construct the mirror quintic, we are ultimately interested in dividing out by the subgroup $\left(\mathbb{Z}_{5}\right)^{3} \subset\left(\mathbb{Z}_{5}\right)^{4}$ fixing $\psi$. This means that we should be looking at orbits of curves under the group $\left(\mathbb{Z}_{5}\right)^{3}$, and a non-trivial stabilizer contributes an additional factor at the very end of the calculation. On the other hand, no subgroup of $S^{5}$ will be gauged, and a curve with non-trivial stabilizer in $S^{5}$ is not special in any other way.

At a more practical level, the phase symmetries act diagonally on the variables parameterizing $A, B$ and $C$, and dividing out by them does not reduce the dimensionality of the problem, but merely the degree (which is quite helpful anyways, of course!). The permutation symmetries act nondiagonally, and can reduce both the dimensionality and the degree. It is a good idea to keep track whether the subgroup of interest acts with unit determinant on the $x_{i}$ 's or not. If it does, one might expect the solutions of the reduced problem to still be isolated, although this is neither necessary nor sufficient in general. Also, we may point out that a conic that is isolated as a solution invariant under a particular symmetry could in fact sit in a family of conics the generic member of which breaks that symmetry.

We will return to pointing out these, and many more, features of the space of conics after we have presented a few explicit solutions.

\section{2. $S^{3}$-invariant conics}

To begin with, one may look for conics that are invariant under permutation of three of the five homogeneous coordinates of $\mathbb{C P}^{4}$, which we choose to be $x_{1}, x_{2}, x_{3}$, see [24]. (If our concern were counting conics, we would of course have to account for that choice.) We parameterize the equations for the plane as follows:

$$
A:\left\{\begin{array}{l}
a_{1}\left(x_{1}+x_{2}+x_{3}\right)+x_{4} \\
a_{2}\left(x_{1}+x_{2}+x_{3}\right)+x_{5}
\end{array}\right\}
$$


and solve for $x_{4}$ and $x_{5}$. Note that this means in principle that we are working in a specific open patch of the full moduli space. One can check that the solutions in the other patches precisely serve to compactify the families that we shall write down below. The conic $B \subset A$ is given by

$$
B:\left\{x_{1}^{2}+x_{2}^{2}+x_{3}^{2}+b_{1}\left(x_{1} x_{2}+x_{1} x_{3}+x_{2} x_{3}\right)\right\}
$$

where we have gauged the coefficient of $x_{1}^{2}+x_{2}^{2}+x_{3}^{2}$ to 1 . This is again only an open patch, but it captures all solutions. For once, we display the residual cubic:

$$
\begin{aligned}
C:\left\{c_{1}\left(x_{1}^{3}+x_{2}^{3}+x_{3}^{3}\right)+c_{2}\left(x_{1}^{2} x_{2}+x_{1}^{2} x_{3}+x_{2}^{2} x_{1}+x_{2}^{2} x_{3}+x_{3}^{2} x_{1}\right.\right. \\
\left.\left.+x_{3}^{2} x_{2}\right)+c_{3} x_{1} x_{2} x_{3}\right\}
\end{aligned}
$$

which depends on three parameters. Finally, there are five symmetric polynomials of degree 5 in three variables, giving rise to as many equations for the six variables $a_{1}, a_{2}, b_{1}, c_{1}, c_{2}$ and $c_{3}$. Thus we see that we generically expect a one-parameter family of solutions (for fixed $\psi$ ). Writing out those equations explicitly, we see that four of them can be solved linearly for $c_{1}, c_{2}, c_{3}$, and $b_{1}$ in terms of $a_{1}$ and $a_{2}$. For example,

$$
b_{1}=\frac{1-2 \psi a_{1} a_{2}}{1-\psi a_{1} a_{2}} \text {. }
$$

The remaining equation is

$$
1-a_{1}^{5}-a_{2}^{5}+5 \psi^{2} a_{1}^{2} a_{2}^{2}-5 \psi a_{1} a_{2}
$$

Thus, for fixed $\psi$, picking any solution of (3.8), the intersection of the quintic $Y_{\psi}$ with the plane (3.4) decomposes as the union of the conic (3.5) and the cubic (3.6). This is the solution found by Mustaţă [24].

For completeness, and anticipating a stratagem that will be relevant later, we note that the invariant ansatz (3.4) is not the only way to produce an $S^{3}$-invariant plane. Indeed, the two equations defining $A$ might also transform in the two-dimensional irreducible representation of $S^{3}$, i.e., $A$ might be given by $\left\{x_{1}-x_{2}, x_{2}-x_{3}\right\}$. This eliminates any free parameters in $A$, while bringing back those in $B$ and $C$ to $16-1=15$, and the number of equations to 21 . In the present case, allowing the equations to transform non-trivially under the symmetry group does not uncover any new solutions. In later examples it will. 


\section{3. $\mathbb{Z}_{2} \times \mathbb{Z}_{2}$-invariant conics}

The next case of interest is the subgroup $\mathbb{Z}_{2} \times \mathbb{Z}_{2} \subset S^{5}$, with generators acting by exchanging $\left(x_{1}, x_{3}\right)$ and $\left(x_{2}, x_{4}\right)$ respectively. ${ }^{2}$ Assuming the equations for the plane to be invariant leads to the ansatz

$$
A:\left\{\begin{array}{l}
x_{1}+x_{3}+a_{1} x_{5} \\
x_{2}+x_{4}+a_{2} x_{5}
\end{array}\right\} .
$$

We here see immediately that for any value of $a_{1}, a_{2}$, the plane (3.9) contains, at $x_{5}=0$, one of the 375 isolated lines discussed in the previous section. Therefore, if the quintic has any conic in such a plane, the residual cubic in Equation (3.1) will be reducible, so that there are then, actually, two conics in that plane. A priori, we do not expect to find any such conic at all, since the quartic curve residual to the line in the plane would need to develop four nodes where we only have two parameters at our disposal to move the plane. The symmetries help, however, as we shall see presently.

We solve the Equations in (3.9) for $x_{1}$ and $x_{2}$, and make the ansatz

$$
b_{6} x_{3}^{2}+b_{5} x_{3} x_{4}+b_{4} x_{3} x_{5}+b_{3} x_{4}^{2}+b_{2} x_{4} x_{5}+b_{1} x_{5}^{2},
$$

for the equation defining the conic. This is invariant under $x_{3} \rightarrow x_{1}=-x_{3}-$ $a_{1} x_{5}$ and under $x_{4} \rightarrow x_{2}=-x_{4}-a_{2} x_{5}$, precisely if

$$
b_{5}=0, \quad b_{2}=a_{2} b_{3}, \quad b_{4}=a_{1} b_{6}
$$

Eliminating the cubic, we find that the solution of (3.1) is, in the gauge $b_{6}=1$, reduced to the three equations

$$
\begin{aligned}
1-a_{1}^{5}-a_{2}^{5}+5 a_{1}^{3} b_{1}-5 a_{1} b_{1}^{2} & =0 \\
a_{2}^{3}-\psi b_{1}-a_{1}^{3} b_{3}+2 a_{1} b_{1} b_{3} & =0 \\
a_{2}-\psi b_{3}+a_{1} b_{3}^{2} & =0
\end{aligned}
$$

for the four variables $a_{1}, a_{2}, b_{1}, b_{3}$. We see that this describes two oneparameter families of conics for each $\psi$ : the first equation admits two solutions for $b_{1}$, the middle equation then determines $b_{3}$ uniquely, while the third equation relates $a_{2}$ and $a_{1}$. The two families share the planes, but not any conics. As in the previous subsection, the Equation (3.12) describes only an open patch of the families. Below, we will see a bit of the compactification,

\footnotetext{
${ }^{2} \mathbb{Z}_{2} \times \mathbb{Z}_{2}$-invariant conics at $\psi=0$ have also been studied in [24].
} 
as dictated by the embedding in the moduli space of conics in $\mathbb{P}^{4}$. As an example, one might verify the symmetry under exchange of $a_{1}$ and $a_{2}$.

\subsection{Taking advantage of phase symmetries}

Going slowly enough over the previous discussion reveals an option for finding further solutions: not all of the $\mathbb{Z}_{2} \times \mathbb{Z}_{2}$ symmetry group under which the plane (3.9) is invariant need to fix the two conics in that plane individually. Instead, the two conics might be exchanged by one generator, and fixed by the other. In particular, we can choose the diagonal $\mathbb{Z}_{2}^{+} \subset \mathbb{Z}_{2} \times \mathbb{Z}_{2}$ to fix the two conics, and the exchange of $\left(x_{1}, x_{3}\right)$ to exchange them. The generator of $\mathbb{Z}_{2}^{+}$acts as

$$
\left(x_{1}, x_{2}, x_{3}, x_{4}, x_{5}\right) \mapsto\left(x_{3}, x_{4}, x_{1}, x_{2}, x_{5}\right) .
$$

This relaxes the constraint (3.11) to

$$
b_{2}=a_{2} b_{3}+\frac{1}{2} a_{1} b_{5}, \quad b_{4}=a_{1} b_{6}+\frac{1}{2} a_{2} b_{5} .
$$

To simplify the equations further, we employ a device that will be useful also later: note that the ansatz (3.9) is covariant under a $\left(\mathbb{Z}_{5}\right)^{2}$ subgroup of the group of phase symmetries, provided we act in a particular way on the coefficients of conic and cubic. So we may absorb those phase symmetries by appropriate variable substitutions, and thereby reduce the degree of the equations. In the present case, we first solve for $b_{1}, b_{2}, b_{3}, b_{4}$, and $a_{2}$ linearly. (Doing this excludes the families above, on which the rank of the equations is reduced.) Still working in the gauge $b_{6}=1$, we then substitute

$$
\psi=\tilde{\psi} a_{1},
$$

and the remaining equations depend only on $a_{1}^{5}$. Introducing $\tilde{a}_{1}=a_{1}^{5}$ as a new variable reduces the degree sufficiently to be able to fully understand the equations. Indeed, $\tilde{a}_{1}$ appears only linearly, and the remaining relations for $b_{5}$ and $\tilde{\psi}$ boil down to

$$
\begin{aligned}
& -64 \tilde{\psi}+2 \tilde{\psi}^{6}-32 b_{5}^{2}+11 \tilde{\psi}^{5} b_{5}^{2}+25 \tilde{\psi}^{4} b_{5}^{4}+30 \tilde{\psi}^{3} b_{5}^{6}+20 \tilde{\psi}^{2} b_{5}^{8} \\
& +7 \tilde{\psi} b_{5}^{10}+b_{5}^{12}=p_{1} p_{2} p_{3}
\end{aligned}
$$


with

$$
\begin{aligned}
p_{1}= & -2+\tilde{\psi}+b_{5}^{2}, \\
p_{2}= & 16+8 \tilde{\psi}+4 \tilde{\psi}^{2}+2 \tilde{\psi}^{3}+\tilde{\psi}^{4}+\left(8+8 \tilde{\psi}+6 \tilde{\psi}^{2}+4 \tilde{\psi}^{3}\right) b_{5}^{2} \\
& +\left(4+6 \tilde{\psi}+6 \tilde{\psi}^{2}\right) b_{5}^{4}+(2+4 \tilde{\psi}) b_{5}^{6}+b_{5}^{8}, \\
p_{3}= & 2 \tilde{\psi}+b_{5}^{2} .
\end{aligned}
$$

We may then substitute back $a_{1}$ and $\psi$, to obtain the relations in a way that will be useful later on. As an example, we write the equations corresponding to $p_{1}$ :

$$
\begin{aligned}
64+5 \psi^{2} a_{1}^{3}-40 \psi a_{1}^{4}+12 a_{1}^{5} & =0 \\
\psi-2 a_{1}+a_{1} b_{5}^{2} & =0 .
\end{aligned}
$$

The two solutions for $b_{5}$ correspond, as it should be, to the two conics that exist in the plane determined by the first equation. The remaining parameters of our ansatz are given by

$$
\begin{gathered}
a_{2}=a_{1}, \quad b_{1}=\frac{1}{8}\left(-\psi a_{1}+6 a_{1}^{2}+2 a_{1}^{2} b_{5}\right), \\
b_{2}=a_{1}+\frac{1}{2} a_{1} b_{5}, \quad b_{3}=1, \quad b_{4}=a_{1}+\frac{1}{2} a_{1} b_{5}, \quad b_{6}=1 .
\end{gathered}
$$

For future reference, we note that the conics corresponding to (3.18) are, in addition to $\mathbb{Z}_{2}^{+}$, invariant under the group $\mathbb{Z}_{2}^{-}$whose generator acts as

$$
\mathbb{Z}_{2}^{-}:\left(x_{1}, x_{2}, x_{3}, x_{4}, x_{5}\right) \mapsto\left(x_{2}, x_{1}, x_{4}, x_{3}, x_{5}\right)
$$

Indeed, when $a_{2}=a_{1}$, the equations for the plane (3.9) are exchanged under that $\mathbb{Z}_{2}^{-}$, while the conic (3.10) is invariant as $b_{6}=b_{3}$ and $b_{4}=b_{2}$.

For $p_{3}$, the relations analogous to (3.18) are

$$
\begin{aligned}
\psi^{10}+4096 a_{1}^{5}-160 \psi^{5} a_{1}^{5}+1024 a_{1}^{10} & =0, \\
2 \psi+a_{1} b_{5}^{2} & =0,
\end{aligned}
$$

and the explicit solution is

$$
\begin{gathered}
a_{2}=\frac{\psi^{2}}{4 a_{1}}, \quad b_{1}=-\frac{\psi^{5}}{64 a_{1}^{3}}+\frac{a_{1}^{2}}{2}+\frac{1}{16} \psi^{2} b_{5} \\
b_{2}=-\frac{\psi^{3}}{8 a_{1}^{2}}+\frac{1}{2} a_{1} b_{5}, \quad b_{3}=-\frac{\psi}{2 a_{1}}, \quad b_{4}=a_{1}+\frac{\psi^{2} b_{5}}{8 a_{1}}, \quad b_{6}=1 .
\end{gathered}
$$

These solutions are invariant only under $\mathbb{Z}_{2}^{+}$. 
Anticipating some of the later discussion, we note that the conic (3.10) is reducible when the $b_{i}$ 's take the values in (3.22), provided $b_{5}$ satisfies the condition in (3.21). So we see that in fact, the plane (3.9) with $4 a_{2} a_{1}=\psi^{2}$ and $a_{1}$ satisfying the first equation in (3.21) meets the quintic in a collection of five lines.

We have not discussed in detail the conics corresponding to the factor $p_{2}$ in (3.17). In fact, that solution arises from (3.18), (3.19) simply by the phase symmetry acting on $x_{2}$ and $x_{4}$. For instance, one may check that instead of $a_{2}=a_{1}$, merely $a_{2}^{5}=a_{1}^{5}$ holds on that solution.

For completeness, we take a brief look at solutions with $b_{6}=b_{3}=0$ (which was excluded by our above choice of gauge). We find that the only such solutions are

$$
a_{1}=a_{2}=b_{2}=b_{3}=b_{4}=b_{6}=0, \quad b_{5}^{2}=5 \psi b_{1}^{2},
$$

so that we recover the conics studied in [1].

\section{5. $\mathbb{Z}_{2}^{-}$-invariant conics}

An important feature of the previous subsection was that the group $\mathbb{Z}_{2}^{+}$(see Equation (3.13)) acts with unit determinant on $\left(x_{1}, x_{2}, x_{3}, x_{4}\right.$ and $\left.x_{5}\right)$, and the number of equations matched the number of variables also in the reduced problem. We were able to fully reduce those equations, and thereby isolate

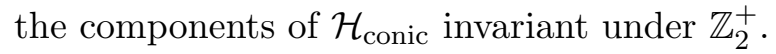

As an ultimate possibility, we now study conics that are invariant under the action

$$
\mathbb{Z}_{2}^{-}:\left(x_{1}, x_{2}, x_{3}, x_{4}, x_{5}\right) \mapsto\left(x_{2}, x_{1}, x_{4}, x_{3}, x_{5}\right)
$$

As symmetries of $\mathbb{P}^{4}$, the groups $\mathbb{Z}_{2}^{+}$and $\mathbb{Z}_{2}^{-}$are of course equivalent. But, as it turns out, we get a new class of solutions if we modify the ansatz for the plane $A$ containing the conic, and take one equation to be invariant, and the other to transform with a sign (this being equivalent to the way (3.20) acted on (3.9)):

$$
A:\left\{\begin{array}{c}
a_{1}\left(x_{1}+x_{2}\right)+a_{2}\left(x_{3}+x_{4}\right)+x_{5} \\
\left(x_{1}-x_{2}\right)+a_{3}\left(x_{3}-x_{4}\right)
\end{array}\right\} .
$$


The equation for the conic has to be invariant (we eliminate only $x_{5}$ in order to make the symmetry manifest):

$$
\begin{aligned}
B: & \left\{b_{1}\left(x_{1}+x_{2}\right)^{2}+b_{2}\left(x_{1}+x_{2}\right)\left(x_{3}+x_{4}\right)+b_{3}\left(x_{3}+x_{4}\right)^{2}\right. \\
& \left.+b_{4}\left(x_{3}-x_{4}\right)^{2}\right\} .
\end{aligned}
$$

Now absorb the phase symmetries by substituting

$$
\begin{gathered}
a_{1} \rightarrow a_{1}^{-1 / 5}, \quad a_{2} \rightarrow a_{2}^{-1 / 5}, \quad a_{3} \rightarrow a_{3} a_{1}^{1 / 5} a_{2}^{-1 / 5}, \quad \psi \rightarrow \psi a_{1}^{-2 / 5} a_{2}^{-2 / 5}, \\
b_{1} \rightarrow b_{1} a_{1}^{-2 / 5}, \quad b_{2} \rightarrow b_{2} a_{1}^{-1 / 5} a_{2}^{-1 / 5}, \quad b_{3} \rightarrow b_{3} a_{2}^{-2 / 5}, \quad b_{4} \rightarrow b_{4} a_{2}^{-2 / 5},
\end{gathered}
$$

where we denote the new variables by the same letters as the old ones. Then, in the patch $b_{2}=1$, the $a_{1}, a_{2}, a_{3}^{2}, b_{4}$ can be solved for linearly. When substituted back, we remain with two equations of relatively high degree involving $b_{1}, b_{3}$ and $\psi$,

$$
q_{1}\left(b_{1}, b_{3}, \psi\right)=q_{2}\left(b_{1}, b_{3}, \psi\right)=0 .
$$

These can be further reduced if we exploit the inherent symmetry of (3.26) exchanging $b_{1}$ and $b_{3}$, and substitute $b_{1}-b_{3}=u, b_{1} b_{3}=v$. Then, we eliminate $\psi$ by computing the resultant of those two equations, to decompose the set of conics invariant under the $\mathbb{Z}_{2}^{-}$-symmetry (3.24) as much as possible into constituents:

$\operatorname{Resultant}\left(q_{1}, q_{2} ; \psi\right)(u, v) \propto(9-12 u+16 v) \cdot(1-4 v) \cdot\left(u^{2}-4 v\right) \cdot Q_{m}$.

Here we have excluded factors that do not lead to a solution of the original system, because the equations for $a_{1}, a_{2}, a_{3}$ and $b_{4}$ that we solved earlier actually became singular. To each factor of (3.29), there corresponds a component ${ }^{3}$ of $\mathcal{H}_{\text {conics }}$ that can be reconstructed in the following way: given a pair $(u, v)$ for which that factor vanishes, we find a $\psi$ solving Equations (3.28) (the existence of a common root of $q_{1}$ and $q_{2}$ being precisely the characterization of the resultant), and then a unique set of $a_{1}, a_{2}, a_{3}^{2}$ and $b_{4}$ solving the equations for a conic on the quintic under the $\mathbb{Z}_{2}^{-}$-invariant ansatz (3.25), (3.26), after absorbing the $\mathbb{Z}_{5} \times \mathbb{Z}_{5}$ phase symmetries as in (3.27). Undoing that substitution introduces two fifth roots of unity, one of

\footnotetext{
${ }^{3}$ We are not claiming here that all of those components are irreducible. It is just the best we can do at this point.
} 
which corresponds to the phase of $\psi$ that originally parameterized the family of quintics, while the other is a genuine label of a conic contained therein. We thus obtain various branches of conics for each factor of the resultant (3.29), depending on which solution we choose. These branches will interact in various ways as (the original) $\psi$ (appearing in (2.1)) is varied around the moduli space. The different factors of (3.29) might split further under this procedure (but they will not mix). An obvious splitting arises when we remember that the map $\left(b_{1}, b_{3}\right) \rightarrow(u, v)$ is actually two-to-one. For example,

$$
9-12 u+16 v=\left(3-4 b_{1}\right)\left(3-4 b_{3}\right),
$$

so that the first factor in (3.29) actually describes two sets of conics in that sense. Also, although we did not bother pointing this out, it is clear that the equations are invariant under $a_{3} \rightarrow-a_{3}$, so we also need to choose a sign for $a_{3}$ when we go back.

The main component of $\mathcal{H}_{\text {conics }}$ that we found is characterized by the factor,

$$
\begin{aligned}
Q_{\mathrm{m}}= & -140544+1312896 u-6157536 u^{2}+20560128 u^{3}-55739073 u^{4} \\
& +126082635 u^{5}-240562314 u^{6}+389983296 u^{7}-517794816 u^{8} \\
& +526312752 u^{9}-386382096 u^{10}+195989568 u^{11}-64755264 u^{12} \\
& +12180480 u^{13}-890112 u^{14}+3526016 v-35327360 u v \\
& +164085512 u^{2} v-490389848 u^{3} v+1119877362 u^{4} v-2054126078 u^{5} v \\
& +2822178044 u^{6} v-2674914608 u^{7} v+1703155648 u^{8} v-783769296 u^{9} v \\
& +331207776 u^{10} v-160872256 u^{11} v+74273920 u^{12} v-20820224 u^{13} v \\
& +1915392 u^{14} v-12887824 v^{2}+156888240 u v^{2}-794842896 u^{2} v^{2} \\
& +1924669488 u^{3} v^{2}-1861954446 u^{4} v^{2}-560979783 u^{5} v^{2} \\
& +2742716878 u^{6} v^{2}-2532259552 u^{7} v^{2}+1357646032 u^{8} v^{2} \\
& -522831968 u^{9} v^{2}+146600816 u^{10} v^{2}-62851072 u^{11} v^{2}+15982784 u^{12} v^{2} \\
& +5083904 u^{13} v^{2}-966912 u^{14} v^{2}-110365024 v^{3}+1349538976 u v^{3} \\
& -5573477584 u^{2} v^{3}+9890213496 u^{3} v^{3}-8559117395 u^{4} v^{3} \\
& +4959540898 u^{5} v^{3}-3410214400 u^{6} v^{3}+1529015152 u^{7} v^{3} \\
& +33207472 u^{8} v^{3}-335159488 u^{9} v^{3}+398796352 u^{10} v^{3}-199492096 u^{11} v^{3} \\
& +27038976 u^{12} v^{3}+206336 u^{13} v^{3}-181248 u^{14} v^{3}-1058031072 v^{4}
\end{aligned}
$$




$$
\begin{aligned}
& +7328123536 u v^{4}-16155350056 u^{2} v^{4}+17717423024 u^{3} v^{4} \\
& -18250232092 u^{4} v^{4}+21436831296 u^{5} v^{4}-16619578848 u^{6} v^{4} \\
& +8429844448 u^{7} v^{4}-3758257792 u^{8} v^{4}+1108256896 u^{9} v^{4} \\
& -254268672 u^{10} v^{4}+129069056 u^{11} v^{4}-52310016 u^{12} v^{4}+4696064 u^{13} v^{4} \\
& +135168 u^{14} v^{4}-1271515824 v^{5}-3515100512 u v^{5}+23558245664 u^{2} v^{5} \\
& -33532680832 u^{3} v^{5}+15994006832 u^{4} v^{5}+1748284832 u^{5} v^{5} \\
& -6786182656 u^{6} v^{5}+5719888128 u^{7} v^{5}-2496033024 u^{8} v^{5} \\
& +544198656 u^{9} v^{5}-76355584 u^{10} v^{5}+61018112 u^{11} v^{5}+3694592 u^{12} v^{5} \\
& -1777664 u^{13} v^{5}+8485369664 v^{6}-39975494784 u v^{6} \\
& +76393384256 u^{2} v^{6}-82428927744 u^{3} v^{6}+64625199040 u^{4} v^{6} \\
& -46320419072 u^{5} v^{6}+28977470976 u^{6} v^{6}-13398732800 u^{7} v^{6} \\
& +5270946816 u^{8} v^{6}-1532405760 u^{9} v^{6}+165781504 u^{10} v^{6} \\
& -21012480 u^{11} v^{6}+2473984 u^{12} v^{6}+3324777728 v^{7}-4295229696 u v^{7} \\
& -21543773440 u^{2} v^{7}+57614347264 u^{3} v^{7}-56924167424 u^{4} v^{7} \\
& +37388443136 u^{5} v^{7}-22467149824 u^{6} v^{7}+7877509120 u^{7} v^{7} \\
& -2088861696 u^{8} v^{7}+451772416 u^{9} v^{7}-78036992 u^{10} v^{7}+6029312 u^{11} v^{7} \\
& -8267872256 v^{8}+34670743552 u v^{8}-42630860800 u^{2} v^{8} \\
& +7651102720 u^{3} v^{8}+12061375488 u^{4} v^{8}-7382695936 u^{5} v^{8} \\
& +6715727872 u^{6} v^{8}-2138890240 u^{7} v^{8}+730972160 u^{8} v^{8} \\
& -79429632 u^{9} v^{8}+8498114560 v^{9}-35617423360 u v^{9} \\
& +49886576640 u^{2} v^{9}-22319595520 u^{3} v^{9}-1981624320 u^{4} v^{9} \\
& +2571264000 u^{5} v^{9}-2668953600 u^{6} v^{9}+344719360 u^{7} v^{9} \\
& -2792865792 v^{10}+10876387328 u v^{10}-12904677376 u^{2} v^{10} \\
& +2578120704 u^{3} v^{10}+3163045888 u^{4} v^{10}-473956352 u^{5} v^{10} \\
& +320798720 v^{11}-891617280 u v^{11}+732364800 u^{2} v^{11}-87818240 u^{3} v^{11}
\end{aligned}
$$

\subsection{Relationships}

We now describe how the solutions of (3.1) that we have found so far by imposing certain symmetries fit together as components of the Hilbert scheme, $\mathcal{H}_{\text {conics }}$, of conics on the one-parameter family of quintics $(2.1)$. (As emphasized before, we do not claim that we have identified all components, nor that all components that we have found are irreducible.) 
3.6.1. $\mathbb{Z}_{2} \times \mathbb{Z}_{2}$ meets $S^{3}$. First of all, we point out that the family of $S^{3}$-invariant conics found by Mustaţă and the family of $\mathbb{Z}_{2} \times \mathbb{Z}_{2}$-invariant conics (3.12) meet. A common member occurs in the first family if we put $a_{1}=1, a_{2}=0$ in (3.4), where the conic acquires some additional symmetry, and in particular the $\mathbb{Z}_{2} \times \mathbb{Z}_{2}$ symmetry manifest in (3.9). (Note that this solves (3.8) and that the conic (3.5) is also invariant because $b_{1}=1$.) To, conversely, exhibit that conic on the $\mathbb{Z}_{2} \times \mathbb{Z}_{2}$-invariant family, we first need to go to a different patch of the moduli space. We note that the plane in (3.9) is equivalent to

$$
\left\{\begin{array}{c}
x_{1}+x_{3}+\tilde{a}_{1}\left(x_{2}+x_{4}\right) \\
\tilde{a}_{2}\left(x_{2}+x_{4}\right)+x_{5}
\end{array}\right\}
$$

where $\tilde{a}_{1}=-a_{1} / a_{2}, \tilde{a}_{2}=a_{2}^{-1}$. If we now put $\tilde{a}_{1}=1, \tilde{a}_{2}=0$, we recover the plane invariant under both $S^{3}$ and $\mathbb{Z}_{2} \times \mathbb{Z}_{2}$ symmetry that we just discussed. The quintic meets that plane in a conic plus three lines. One of those is the line common to all the planes, the irreducible conic is the $S^{4}$-enhancement in one of the families of $\mathbb{Z}_{2} \times \mathbb{Z}_{2}$-invariant conics, while the remaining two lines represent the second family.

Another example of a common member of the two types of families occurs when $\tilde{a}_{1}=0, \tilde{a}_{2}=1$ in (3.32). This conic appears on the family invariant under permutation of $\left(x_{2}, x_{4}, x_{5}\right)$ in the limit $a_{2}=a_{1}, a_{1}^{-1}=0$ in the appropriate version of the plane (3.4).

It is a useful exercise to write the equations for the families of $\mathbb{Z}_{2} \times \mathbb{Z}_{2^{-}}$ invariant conics in the patch with coordinates $\tilde{a}_{1}, \tilde{a}_{2}$. The parameterization (3.10) was valid as long as we could eliminate $x_{1}$ and $x_{2}$ - this is not possible when $\tilde{a}_{2} \rightarrow 0$ in (3.32). So eliminating $x_{5}$ instead of $x_{2}$, we write for the conic

$$
\tilde{b}_{1} x_{2}^{2}+\tilde{a}_{1} \tilde{b}_{3} x_{2} x_{3}+\tilde{b}_{3} x_{3}^{2}+\tilde{b}_{4} x_{2} x_{4}+\tilde{a}_{1} \tilde{b}_{3} x_{3} x_{4}+\tilde{b}_{1} x_{4}^{2} .
$$

Then, in the patch $\tilde{b}_{1}=1$, the equations for the family are

$$
\begin{array}{r}
-5 \tilde{a}_{1}+5 \tilde{a}_{1}^{3} \tilde{b}_{3}+\left(1-\tilde{a}_{1}^{5}-\tilde{a}_{2}^{5}\right) \tilde{b}_{3}^{2}=0 \\
-1-4 \tilde{a}_{1}^{5}-4 \tilde{a}_{2}^{5}+\left(1+4 \tilde{a}_{1}^{5}+4 \tilde{a}_{2}^{5}\right) \tilde{b}_{4}+\left(1-\tilde{a}_{1}^{5}-\tilde{a}_{2}^{5}\right) \tilde{b}_{4}^{2}=0 \\
-10 \tilde{a}_{1}^{3}-5 \psi \tilde{a}_{2}+5 \tilde{a}_{1}^{3} \tilde{b}_{4}+\tilde{b}_{3}\left(1+4 \tilde{a}_{1}^{5}+4 \tilde{a}_{2}^{5}\right)+2 \tilde{b}_{3} \tilde{b}_{4}\left(1-\tilde{a}_{1}^{5}-\tilde{a}_{2}^{5}\right)=0
\end{array}
$$

These equations have a structure comparable to that of (3.12), and in the relevant open patches the two systems are completely equivalent. 
3.6.2. Reducible conics. Secondly, we record that the $\mathbb{Z}_{2} \times \mathbb{Z}_{2}$-invariant families contain reducible conics. We have seen this in the discussion we just had at $\tilde{a}_{1}=1, \tilde{a}_{2}=0$. Another example is $\tilde{a}_{1}=\tilde{a}_{2}=0$, which plane, $x_{1}+x_{3}=x_{5}=0$, meets the quintic in the union of five of the 375 isolated lines that we discussed in the previous section. Yet another example is $a_{1}=$ $1, a_{2}=0$ in (3.9).

3.6.3. $\mathbb{Z}_{2}^{-}$meets $\mathbb{Z}_{2}^{+}$. Thirdly, we study in more detail the locus $u^{2}-$ $4 v=\left(b_{1}-b_{3}\right)^{2}=0$ corresponding to the vanishing of the third factor of (3.29) in the set of $\mathbb{Z}_{2}^{-}$-invariant conics. We find that the relevant planes have $a_{2}=a_{1}, a_{3}=-1$ in (3.25), and after eliminating the cubic, the resulting equations split in two components. One solution may be written as

$$
a_{1}=a_{2}=\frac{1}{\psi}, \quad a_{3}=-1, \quad b_{2}=1, \quad b_{3}=b_{1}, \quad b_{4}=-1+2 b_{1}
$$

with $b_{1}$ satisfying

$$
-16+\psi^{5}+64 b_{1}+\psi^{5} b_{1}-64 b_{1}^{2}+4 \psi^{5} b_{1}^{2}=0 .
$$

The other is characterized by the vanishing of

$$
3-20 \psi a_{1}+5 \psi^{2} a_{1}^{2}+512 a_{1}^{5} .
$$

After $a_{1} \rightarrow 1 /\left(2 a_{1}\right)$, we may recognize this equation as being equivalent to the first line in (3.18). Indeed, when $a_{2}=a_{1}$, and $a_{3}=-1$, the ansatz (3.25) is also invariant under $\mathbb{Z}_{2}^{+}$from (3.13), and with appropriate substitutions, the solution given by (3.37) is nothing but that in (3.18), (3.19). One can also check that the solution (3.35), (3.36) is contained in the family (3.12) at $a_{1}=a_{2}=\psi / 2$.

3.6.4. A family of reducible conics. Next, we discuss the conics associated with the vanishing of the second factor in (3.29),

$$
1-4 v=1-4 b_{1} b_{3} .
$$

We see quite rapidly that under this condition, the conic (3.26) is reducible (remember that we work with $b_{2}=1$ ). The two components must be lines on the mirror quintic, which are completely understood as reviewed in the previous section. Since it is clear that the relevant lines are not on the list of 375 isolated lines, they must belong to the families containing the van Geemen lines. This indeed makes sense: for generic $\psi$, we have two distinct 
families of lines in $Y_{\psi}$ that meet in a curve $K_{\psi} \subset Y_{\psi}$. Each point in $K_{\psi}$ is the intersection point of two lines, one from each family, which together can be properly viewed as a reducible conic. (The part of the Clemens conjecture stating that rational curves are generically disjoint obviously does not hold here.) In other words, each $Y_{\psi}$ contains a family of reducible conics parameterized by $K_{\psi}$. At certain isolated points in $K_{\psi}$, the reducible conic acquires the $\mathbb{Z}_{2}^{-}$symmetry we have discussed, and shows up on our list.

As a further check, one may start from the $\mathbb{Z}_{2}^{-}$-invariant solutions with $1-4 v=0$ and verify that it indeed deforms as a one-parameter family of reducible conics, which generically break the $\mathbb{Z}_{2}^{-}$symmetry.

Moreover, we can now remember the $\mathbb{Z}_{2}^{+}$-invariant solution (3.21), (3.22), which conics were also reducible with components not on the list of 375 . These must also belong to the family of reducible conics parameterized by $K_{\psi}$. In fact, they must correspond to singular points on $K_{\psi}$ since we actually have four such lines (i.e., two reducible conics) in the corresponding planes.

3.6.5. Another coincidence. Finally, we note that the conics associated with the vanishing of the first factor in (3.29), which according to (3.30) can be realized for instance at $b_{1}=3 / 4$, actually belong to the Mustaţă family of $S^{3}$-invariant conics. This can easily be checked.

\section{Summary so far}

We have seen that the generic $Y_{\psi}$ contains (at least) three types of families of conics, and we have identified a number of isolated conics. We have not attempted to enumerate the solutions, mostly because we did not work out the global description of all the families. Numerical methods indicate that these might in fact be all solutions: elementary search algorithms (such as those of Mathematica) return only solutions on one of our families, or isolated solutions with a non-trivial symmetry group. (This has to be taken with a dose of skepsis, because such algorithms have a higher chance of finding solutions with symmetry or those occurring in families.) We have also checked that there are no solutions with other types of symmetry enhancements than those we have discussed.

There is first of all the family with $S^{3}$ symmetry, parameterized by solutions of (3.8), and originally found by Mustaţă [24]. Secondly, we found two families of conics invariant under the action of a $\mathbb{Z}_{2} \times \mathbb{Z}_{2}$ symmetry group. These are parameterized by solutions of (3.12), and have the interesting feature of sharing their planes. The $\mathbb{Z}_{2} \times \mathbb{Z}_{2}$ invariant families meet the $S^{3}$ invariant families in various ways, and contain reducible conics. Thirdly, 
there is a family consisting entirely of reducible conics. This family can be obtained by intersecting the van Geemen families of lines. We have not written down the equations describing that family globally, but identified two members, one with $\mathbb{Z}_{2}^{+}$symmetry (3.21) and one with $\mathbb{Z}_{2}^{-}$symmetry (3.38).

Turning to the isolated conics, we have first of all those studied in [1], see Equation (3.23). Secondly, we have the $\mathbb{Z}_{2}^{+} \times \mathbb{Z}_{2}^{-}$-invariant conics of (3.18), (3.19) (their $\mathbb{Z}_{5}$-orbit was also discussed around there). One may check (for instance numerically), that these conics are in fact isolated also in the space of conics without any discrete symmetries. Finally, we have $\mathbb{Z}_{2}^{-}$-invariant conics associated with solutions of (3.31), which are also isolated forgetting the symmetry. As it stands, (3.31) is not particularly useful of course. It is somewhat unfortunate that we have not been able to make further progress on those equations, for instance with the purpose of checking whether the corresponding part of $\mathcal{H}_{\text {conics }}$ is in fact irreducible or not. In the next section, we will retreat to studying the expansion in the large complex structure limit.

We now switch to the main topic of interest in this paper, namely the Abel-Jacobi image of $\mathcal{H}_{\text {conics. }}$. We first of all dispose of the families: recall that the isolated lines have a vanishing Abel-Jacobi image (in the sense described in the introduction, and in Section 2), and the van Geemen lines give rise to the inhomogeneity (2.12). As described above, the families are all algebraically equivalent to some combination of those lines, and therefore they do not give rise to any new inhomogeneity.

We only remain with the isolated conics. We shall denote the family of conics (component of $\mathcal{H}_{\text {conics }}$ ) studied in [1], $\mathcal{I}_{0}$. That associated with (3.18) will be called "the first component", $\mathcal{I}_{1}$, and that of (3.31), (which might still be reducible), the "main component", $\mathcal{I}_{2}$.

\section{Expansion in large complex structure limit}

We realized in the previous section that the expression (3.31) is too large to allow writing down explicitly all coefficients determining the "main component", $\mathcal{I}_{2}$, of the space of $\mathbb{Z}_{2}^{-}$-invariant conics, at least not without significantly increased computing power. Progress is still possible, however.

\subsection{Newton-Puiseux expansion}

The main idea is easy to describe: instead of reducing the equations satisfied by the parameters in (3.25), (3.26) algebraically, we expand those parameters in (fractional) power series around large complex structure point $\psi=\infty$, and determine the expansion coefficients recursively from the equations. This is 
in principle sufficient to calculate the expansion of $\mathcal{W}(z)$ for the purpose of testing mirror symmetry. In practice, the calculation is limited to the first few orders in the expansion. The method will also not allow to easily calculate monodromies around complex structure moduli space, which would be desirable in order to fix the solution of the homogeneous Picard-Fuchs equation in $\mathcal{W}$.

For what we will call the "first component" of $\mathcal{H}_{\text {conics }}, \mathcal{I}_{1}$, we are able to calculate the inhomogeneity exactly, see (5.27). This allows expansion to much higher order, calculation of monodromies, and is also a useful crosscheck on the calculations around $\mathcal{I}_{2}$.

But let us first be a bit more general and take $v$ as any one of the parameters entering an ansatz for the curve $\mathcal{C} \subset \mathcal{Y}$ under consideration (e.g., one of the $a_{i}$ or $b_{j}$ in (3.25), (3.26)). (We could also imagine $\mathcal{Y}$ to be a more general family of algebraic varieties than the mirror quintic, degenerating in some way, and $\mathcal{C}$ to be some general algebraic cycle.) For a one-parameter family, $v$ will, as a function of $\psi$, satisfy a parameter-dependent polynomial equation

$$
P(v, \psi)=0
$$

(obtained in the example by projecting Equation (3.1) onto $(v, \psi)$, and generally at least as complicated as (3.31)). Let's assume that $P$ is irreducible.

Following Newton, we can study the behavior of the roots of (5.1) as $\psi \rightarrow \infty$, by looking at the polygon spanned by monomials with non-zero coefficients in $P(v, \psi)$ : say

$$
P(v, \psi)=\sum_{m, n} p_{m, n} v^{m} \psi^{n},
$$

and let $\Pi$ be the convex hull of points $(m, n) \in \mathbb{Z}^{2}$ with $p_{m, n} \neq 0$. Because we are looking at $\psi \rightarrow \infty$, the interesting part of $\Pi$ actually is its upper boundary, $\widehat{\Pi}$, which is the set of point $(m, n) \in \Pi$ such that $\left(m, n^{\prime}\right) \notin \Pi$ for $n^{\prime}>n$. This $\widehat{\Pi}$ consists of a finite sequence of segments of decreasing slope and varying length.

Let $v_{k}(\psi)$ (with $1 \leq k \leq E$ ) be one of the $E$ roots of $P(v, \psi)=0$ for fixed $\psi$. (Here, $E:=\max \left\{m, p_{m, n} \neq 0\right.$ for some $\left.n\right\}$ is the $v$-degree of $P$.). There is then a rational number $\alpha_{k}$ such that

$$
c_{0}:=\lim _{\psi \rightarrow \infty} \psi^{-\alpha_{k}} v_{k}(\psi) \notin\{0, \infty\}
$$


In other words, we are making an ansatz of the form

$$
v_{k}(\psi)=\psi^{\alpha_{k}}\left(c_{0}+\mathcal{O}\left(\psi^{-\beta}\right)\right) \quad \text { with } c_{0} \neq 0 \text { and } \beta>0
$$

plug this into (5.2), and collect terms of same order in $\psi$ :

$$
P\left(v_{k}(\psi), \psi\right)=\psi^{\alpha_{P}}\left(P_{0}\left(c_{0}\right)+\mathcal{O}\left(\psi^{-\beta}\right)\right) .
$$

Then,

$$
\alpha_{P}=\max \left\{\alpha_{k} m+n, p_{m, n} \neq 0\right\},
$$

and

$$
P_{0}\left(c_{0}\right)=\sum_{\alpha_{k} m+n=\alpha_{P}} p_{m, n} c_{0}^{m}
$$

For $P_{0}\left(c_{0}\right)=0$ to have a non-zero solution, there must be at least two nonzero terms in $P_{0}\left(c_{0}\right)$. This shows that (i) $\alpha_{k}$ must be the negative slope of one of the upper edges of $\Pi$ (i.e., of a segment of $\widehat{\Pi}$ ), and (ii) $P_{0}\left(c_{0}\right)$ is the sum of $p_{m, n} c_{0}^{m}$ along that segment. In particular, the degree of $P_{0}\left(c_{0}\right)$ is the length of (the projection onto the $m$-axis of) that segment.

Having found the lowest order term, we can proceed with the expansion (5.4) to higher order. Several things can happen: for example, the polynomial $P_{0}\left(c_{0}\right)$ might be reducible, or the sub-leading terms in (5.5) might vanish together with $P_{0}\left(c_{0}\right)$. The next term in (5.5) that does not vanish after imposing the leading order equation will determine the exponent $\beta$ in (5.4). Past this, and if the original $P$ is irreducible, we are set to calculate the coefficients in

$$
v_{k}(\psi)=\psi^{\alpha_{k}} \sum_{d=0}^{\infty} c_{d} \psi^{-\beta d}
$$

recursively from the $\psi$-expansion (5.5). It is in the nature of things that the coefficients $c_{d}$ for $d>1$ are finite algebraic expressions in $c_{0}$, modulo $P_{0}\left(c_{0}\right)=0$.

In a somewhat more formal language, we can view $v$ as generator of an algebraic extension of the field of rational functions on $\psi$-space, of degree $E$. When localizing that extension at $\psi=\infty$, it splits into extensions of the local field of power (Laurent) series in $\psi^{-1}$, of degree $e_{k}$ given by the length of the corresponding segment of $\widehat{\Pi}$. (Observe that $\sum e_{k}=E$.) The generators of these local extensions are precisely the Puiseux series (5.8). 
Geometrically, we think of an algebraic curve (5.1) as an $E$-fold cover of $\psi$ space, and what we are doing is simply parameterizing the various branches at $\psi=\infty$.

An underlying piece of structure is hidden in the following fact: the coefficients $p_{m, n}$ are, generally speaking, algebraic combinations of the coefficients entering the definition of $\mathcal{Y}$ (and, possibly, the ansatz for $\mathcal{C}$ ). In other words, the $p_{m, n}$ live in the field $K$ over which the underlying algebraic variety (and, possibly, the ansatz for $\mathcal{C}$ ) is defined. As we have seen above, to any branch of (5.1) is associated a polynomial $P_{0} \in K\left[c_{0}\right]$ whose vanishing determines the leading coefficient $c_{0}$, and all other coefficients are algebraic combinations of $c_{0}$. Thus, each branch belongs to a certain algebraic extension $K\left(c_{0}\right)$ of the residue field $K$ at $\psi=\infty$.

Given this structure, we now think of the branches of solutions of (5.1) with the same leading exponent and extension of residue field (splitting $P_{0}$ into irreducible factors if necessary) together in one group. It should be kept in mind, however, that $P_{0}$ does not fully characterize the local extension because as discussed above, sub-leading terms in $P(v, \psi)$ could play a significant role. And the global extension determined by $P(v, \psi)$ itself of course is not visible in the local expansion of any given group.

When we do not have the power to reduce to an equation of the type (5.1) explicitly, we can still obtain the Puiseux expansion of the various parameters by studying the original larger system of Equations (3.1). Assuming the underlying family to be one-dimensional, the discussion is similar, with Newton polygon replaced by Newton polyhedron, and vectors of leading exponents. The comments about importance of sub-leading terms in the expansion of the equations however become more acute. Indeed, with more equations, there are more ways in which they can be degenerate, and the actual extension of $K$ governing each group of solutions might only be determined at higher order in the expansion.

There are several more possibilities with higher-dimensional families: for example, a family of cycles could define a transcendental extension of the parameter space, or require an additional blowup on top of the extension.

We also mention an interesting alternative point of view on the expansion we have discussed: so far, we started with curves embedded in $\mathbb{P}^{4}$, and imagined solving the equations that determined which of those would lie in the quintic hypersurface, then passing to the limit $\psi \rightarrow \infty$. Instead, we might also first go to large complex structure, and note that the mirror quintic degenerates there into the union of five copies of $\mathbb{P}^{3}$. While curves on the quintic are virtually rigid, curves in projective space have a large number of parameters. So the problem to study is the lifting of these moduli spaces 


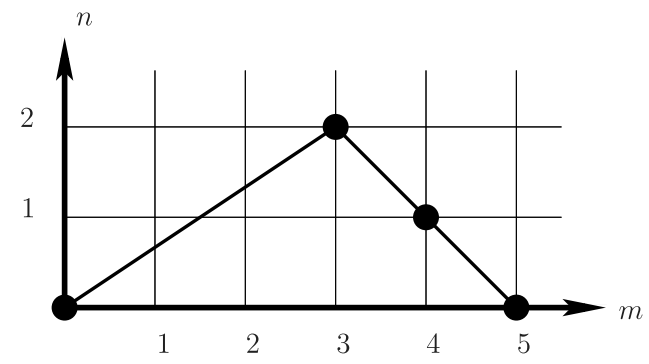

Figure 1: Newton polygon of the Equation (5.9) for the first component of Hilbert scheme of conics on the mirror quintic. A full circle means that the coefficient of the corresponding monomial $a^{m} \psi^{n}$ does not vanish.

under the perturbation away from large complex structure. This description is more intrinsic and presumably better suited to understand the mirror symmetry.

Finally, we note that in our example, the initial family $\mathcal{Y}$ is defined of course over $K=\mathbb{Q}$. In fact, it is not entirely clear how to imagine families of Calabi-Yau manifolds defined intrinsically over a number field other than the rationals, other than by specializing parameters of a higher-dimensional family. ${ }^{4}$ With a D-brane (in the form of an algebraic cycle) on top of $\mathcal{Y}$, extensions of $\mathbb{Q}$ are forced on us.

\subsection{The first component}

Let's warm-up to Newton-Puiseux expansions on the first component $\mathcal{I}_{1}$ of $\mathcal{H}_{\text {conics }}$, for which we can write down the equations globally in $\psi$. We begin by writing (3.18) in the form

$$
\begin{array}{r}
64+5 a^{3} \psi^{2}-40 a^{4} \psi+12 a^{5}=0, \\
-128-5 a^{2} \psi^{3}+40 a^{3} \psi^{2}-12 a^{4} \psi+64 b^{2}=0,
\end{array}
$$

with the abbreviation $a \equiv a_{1}=a_{2}, b \equiv b_{5}$. Recall again that the vanishing of the first equation selects a plane (3.9) intersecting the quintic in (a line and) two conics determined by (3.10), (3.19), and distinguished by the choice of root in (5.10).

The Newton polygon of (5.9) is shown in figure 1.

We see that in the limit $\psi \rightarrow \infty$, the five branches of solutions of (5.9) split into two groups, with asymptotic exponent for $a$ given by the negative

\footnotetext{
${ }^{4}$ I thank Ron Donagi for a helpful discussion on this issue.
} 
slope of the two upper segments of the Newton polygon: the first group has asymptotic behavior

$$
a=\psi^{-2 / 3} a_{0}+\cdots
$$

with

$$
64+5 a_{0}^{3}=0
$$

while the second,

$$
a=\psi a_{0}+\cdots
$$

with $a_{0}$ one of the roots of the equation

$$
12 a_{0}^{2}-40 a_{0}+5=0
$$

To determine the sub-leading terms, and the expansion of $b$, we plug the leading order solution back into (5.9), (5.10). We find that for the first group

$$
b=b_{0} \psi^{5 / 6}+\cdots
$$

with

$$
64 b_{0}^{2}-5 a_{0}^{2}=0
$$

and the local expansion parameter is $\psi^{-5 / 3}$.

For the second group, with local parameter $\psi^{-5}$, the leading order terms in (5.10) vanish under the condition (5.14). Therefore, we have to determine the leading order term in

$$
b=b_{0}+\cdots
$$

from the sub-leading terms in (5.10):

$$
64+5 a_{0} a_{1}-60 a_{0}^{2} a_{1}+24 a_{0}^{3} a_{1}-32 b_{0}^{2}=0 .
$$

Since this involves the coefficient $a_{1}$ in the expansion of $a$ :

$$
a=a_{0} \psi+a_{1} \psi^{-4}+\ldots
$$


we first have to solve (5.9) to that order. We find

$$
a_{1}=\frac{128\left(2729-852 a_{0}\right)}{425},
$$

and then the equation

$$
30-12 a_{0}+5 b_{0}^{2}=0
$$

In terms of the residue field at $\psi=\infty$, this is a second quadratic extension on top of (5.14).

To write the expansion in a more compact form that we will use later on, we introduce the more convenient

$$
w=\frac{1}{5 \psi}=z^{1 / 5} .
$$

Then, on the first group of branches of (5.9), (5.10), we have

$$
\begin{aligned}
a= & -\frac{4 \lambda^{2}}{5} w^{2 / 3}+\frac{128 \lambda^{4}}{15} w^{7 / 3}-\frac{492800}{3} w^{4}+\frac{534732800 \lambda^{2}}{81} w^{17 / 3} \\
& -\frac{73034301440 \lambda^{4}}{243} w^{22 / 3}+\cdots \\
b= & \frac{\lambda^{5}}{1250} w^{-5 / 6}+\frac{14 \lambda}{3} w^{5 / 6}-\frac{1132 \lambda^{3}}{15} w^{5 / 2}+\frac{1035344 \lambda^{5}}{405} w^{25 / 6} \\
& -\frac{16285375600 \lambda}{243} w^{35 / 6}+\cdots
\end{aligned}
$$

with $\lambda$ one of the roots of the equation,

$$
\lambda^{6}=5^{4}
$$

We might record here a typical feature of these expansions: Equation (5.24) means that $\lambda$ is, up to a root of unity, equal to $5^{2 / 3}$. A third root of unity is equivalent to the phase of the local expansion parameter, $w^{5 / 3}$. The additional choice of sign is associated with the choice of root in (5.10). The local monodromy $w \rightarrow \mathrm{e}^{2 \pi \mathrm{i}} w$ permutes those branches cyclically. (As one might expect, the better local variable is actually $z=w^{5}$. The concomitant fifth roots of unity in a will cancel out in (5.27).) 
For the second group,

$$
\begin{aligned}
a= & \frac{6+\lambda^{2}}{12} w^{-1}-\frac{3200\left(-599+355 \lambda^{2}\right)}{17} w^{4} \\
& +\frac{76800000\left(-23778566+14088349 \lambda^{2}\right)}{289} w^{9}-\ldots, \\
b= & \lambda+\frac{2000\left(-74708 \lambda+44263 \lambda^{3}\right)}{51} w^{5} \\
& -\frac{1600000\left(-326587981456 \lambda+193497180065 \lambda^{3}\right)}{2601} w^{10}+\ldots,
\end{aligned}
$$

where $\lambda$ is one of the roots of

$$
5 \lambda^{4}+20 \lambda^{2}-48=0
$$

Note that while again the choice of sign for $\lambda$ originates from (5.10), the local monodromy $w \rightarrow \mathrm{e}^{2 \pi \mathrm{i}} w$ acts trivially.

Before leaving this family of cycles for a while, we show the result of the computation of the inhomogeneous Picard-Fuchs equation. The algorithm of $[1,2]$ can be applied without much change. The main complication is that one has to keep the parameters $a$ and $b$ implicit throughout. Since the line residual to the two conics has a vanishing superpotential (see Section 2 ), the inhomogeneity should be odd under $b \rightarrow-b$. With standard conventions, such as reviewed in Section 2, we find the Picard-Fuchs inhomogeneity associated with conics in $\mathcal{I}_{1}$ to be:

$$
\begin{aligned}
& \mathcal{L} \int^{C} \Omega=f(z) \\
& f(z)=\frac{1}{4 \pi^{2}} \frac{b}{320\left(-128+3 \psi^{5}\right)^{3}\left(-5308416+26104832 \psi^{5}+459 \psi^{10}\right)^{3}} \cdot \\
& \quad \cdot\left[-3529208202219460015329116160-5917959309462446377556508672 a^{4} \psi\right. \\
& \quad-24080174251679112693326807040 a^{3} \psi^{2}-37102979749413690361774080000 a^{2} \psi^{3} \\
& \quad+5322140674208202106664386560 a \psi^{4}+377013614277474642973792665600 \psi^{5} \\
& \quad-223673316478788106348117622784 a^{4} \psi^{6}+231620425022730366652294103040 a^{3} \psi^{7} \\
& \quad+577173365083785450174157946880 a^{2} \psi^{8}+1161971462867073400022583214080 a \psi^{9} \\
& \quad+1138625829170016488325937889280 \psi^{10}-162426814061060730487566237696 a^{4} \psi^{11} \\
& \quad+462200036747394287493017763840 a^{3} \psi^{12}+196861662250863298084696227840 a^{2} \psi^{13} \\
& \quad-198567289143941889876285194240 a \psi^{14}+385678957625260010043531591680 \psi^{15}
\end{aligned}
$$




$$
\begin{aligned}
& -188475902674373195063233609728 a^{4} \psi^{16}+397300557436660139725013647360 a^{3} \psi^{17} \\
& +468813519263945326185655828480 a^{2} \psi^{18}+479723528675140620247262822400 a \psi^{19} \\
& +352752475928491530510768537600 \psi^{20}-39263076586488037778065981440 a^{4} \psi^{21} \\
& +110777498597321397283848192000 a^{3} \psi^{22}+42233632645599612642734899200 a^{2} \psi^{23} \\
& +16695932913990986817444249600 a \psi^{24}+5506564481958675778539356160 \psi^{25} \\
& -279092702543449176793939968 a^{4} \psi^{26}+884770078321237750123069440 a^{3} \psi^{27} \\
& +34251597272406042397900800 a^{2} \psi^{28}-12180273406238980319477760 a \psi^{29} \\
& -7891860706457745044275200 \psi^{30}+557447463014026659692544 a^{4} \psi^{31} \\
& -1763923787950883886858240 a^{3} \psi^{32}-71223763050638247444480 a^{2} \psi^{33} \\
& +4711857482247092305920 a \psi^{34}+1639504965244195307520 \psi^{35} \\
& -34139433836832735744 a^{4} \psi^{36}+110844573279392655360 a^{3} \psi^{37} \\
& -4645064401757907840 a^{2} \psi^{38}-375748813003714560 a \psi^{39}-14770116391956480 \psi^{40} \\
& +66315921005988 a^{4} \psi^{41}-220588897640760 a^{3} \psi^{42}+26084392488495 a^{2} \psi^{43} \\
& \left.+193405158000 a \psi^{44}\right]
\end{aligned}
$$

We will not say much here about the structure of that result, just as we skipped the detailed discussion of the geometry of $\mathcal{I}_{1}$. Note however that the factor $-128+3 \psi^{5}$ in the denominator indicates an interesting interaction of $\mathcal{I}_{1}$ with the van Geemen lines (cf., Equation (2.12)). It is easy to check that the conics in $\mathcal{I}_{1}$ become reducible there (although not only there). The other factor in the denominator is the discriminant of (5.9).

In the expansion (5.23), (5.27) becomes

$$
\begin{aligned}
4 \pi^{2} f(z)= & \frac{25 \lambda}{54} z^{1 / 6}-\frac{2003 \lambda^{3}}{8} z^{1 / 2}+\frac{18846875 \lambda^{5}}{486} z^{5 / 6} \\
& -\frac{6020738135875 \lambda}{2187} z^{7 / 6}+\cdots
\end{aligned}
$$

and on the second group of branches, (5.25), we have

$$
\begin{aligned}
4 \pi^{2} f(z)= & 10000\left(-7624 \lambda+4517 \lambda^{3}\right) z \\
& -\frac{4000000\left(-520331498984 \lambda+308286536785 \lambda^{3}\right)}{51} z^{2}+\cdots .
\end{aligned}
$$

\subsection{The main component}

We now turn to Puiseux expansions of the solutions of (3.1) that satisfy $Q_{m}=0$ (see Equation (3.31)). The ansatzs for plane and conic are (see 
Equations (3.25), (3.26)),

$$
\begin{aligned}
& A:\left\{\begin{array}{c}
a_{1}\left(x_{1}+x_{2}\right)+a_{2}\left(x_{3}+x_{4}\right)+x_{5} \\
\left(x_{1}-x_{2}\right)+a_{3}\left(x_{3}-x_{4}\right)
\end{array}\right\}, \\
& B:\left\{b_{1}\left(x_{1}+x_{2}\right)^{2}+b_{2}\left(x_{1}+x_{2}\right)\left(x_{3}+x_{4}\right)+b_{3}\left(x_{3}+x_{4}\right)^{2}+b_{4}\left(x_{3}-x_{4}\right)^{2}\right\} .
\end{aligned}
$$

For completeness, we display the ansatz for the cubic,

$$
\begin{aligned}
C: & \left\{c_{1}\left(x_{1}+x_{2}\right)^{3}+c_{2}\left(x_{1}+x_{2}\right)^{2}\left(x_{3}+x_{4}\right)+c_{3}\left(x_{1}+x_{2}\right)\left(x_{3}+x_{4}\right)^{2}\right. \\
& \left.+c_{4}\left(x_{3}+x_{4}\right)^{3}+c_{5}\left(x_{1}+x_{2}\right)\left(x_{3}-x_{4}\right)^{2}+c_{6}\left(x_{3}+x_{4}\right)\left(x_{3}-x_{4}\right)^{2}\right\}
\end{aligned}
$$

as well as the full set of relations,

$$
\begin{array}{ll}
\frac{1}{80}-\frac{a_{1}^{5}}{5}-b_{1} c_{1}, & -\frac{\psi a_{1}}{16}+\frac{a_{3}^{2}}{8}-b_{4} c_{1}-b_{1} c_{5}, \\
-a_{1}^{4} a_{2}-b_{2} c_{1}-b_{1} c_{2}, & -\frac{\psi a_{2}}{16}-b_{4} c_{2}-b_{2} c_{5}-b_{1} c_{6}, \\
\frac{\psi a_{1}}{16}-2 a_{1}^{3} a_{2}^{2}-b_{3} c_{1}-b_{2} c_{2}-b_{1} c_{3}, & -\frac{\psi a_{1} a_{3}^{2}}{16}-b_{4} c_{3}-b_{3} c_{5}-b_{2} c_{6}, \\
\frac{\psi a_{2}}{16}-2 a_{1}^{2} a_{2}^{3}-b_{3} c_{2}-b_{2} c_{3}-b_{1} c_{4}, & \frac{1}{8}-\frac{\psi a_{2} a_{3}^{2}}{16}-b_{4} c_{4}-b_{3} c_{6}, \\
-a_{1} a_{2}^{4}-b_{3} c_{3}-b_{2} c_{4}, & \frac{\psi a_{1} a_{3}^{2}}{16}+\frac{a_{3}^{4}}{16}-b_{4} c_{5}, \\
\frac{1}{80}-\frac{a_{2}^{5}}{5}-b_{3} c_{4}, & \frac{1}{16}+\frac{\psi a_{2} a_{3}^{2}}{16}-b_{4} c_{6} .
\end{array}
$$

After scaling one of the $b_{j}$ 's to 1 , we have 12 equations for 12 variables, in addition to $\psi$, which we want to turn into a local expansion parameter. For each of our variables $v_{i}(i=1, \ldots, 12)$, we make an ansatz of the form

$$
v_{i}=\sum_{d=0}\left(v_{i}\right)_{d} \psi^{\alpha_{i}-\beta d}
$$

with rational $\alpha_{i}, \beta$, plug into those equations, and solve order by order in $\psi .^{5}$

${ }^{5}$ Implementing this requires more diligence and patience than is appropriate to perhaps explain. 
Table 1: Groups of branches of $\mathbb{Z}_{2}^{-}$-invariant conics. Some choices capture fairly obvious symmetries of the ansatz: $a_{3} \mapsto-a_{3}$ corresponds to exchange of $x_{3}$ and $x_{4}$. Multiplication of $\left(x_{1}, x_{2}\right)$ and $\left(x_{3}, x_{4}\right)$ by opposite fifth roots of unity can also be absorbed without touching the local expansion parameter. The exchange $a_{1} \leftrightarrow a_{2}, a_{3} \leftrightarrow 1 / a_{3}$ produces further groups, but leaves the first invariant (this is related to the symmetry $\lambda \rightarrow 1 / \lambda$ ). In each group, $0^{*}$ is the exponent of the variable that we have found convenient to scale to 1 .

\begin{tabular}{|l|l|l|c|}
\hline No. & $\begin{array}{l}\text { Exponents, in order } \\
\left(a_{1}, a_{2}, a_{3}, b_{1}, b_{2}, b_{3}, b_{4}\right)\end{array}$ & $\begin{array}{l}\text { Local } \\
\text { par. }\end{array}$ & $\begin{array}{l}\text { Generator of number field and } \\
\text { minimal polynomial }\end{array}$ \\
\hline & & & $\begin{array}{c}\lambda^{10}-243 \lambda^{9}+27675 \lambda^{8} \\
-1529140 \lambda^{7}+49599473 \lambda^{6}\end{array}$ \\
& $\left(-1,-1,0,0,0,0,0^{*}\right)$ & $\psi^{-5}$ & $\lambda=\left(a_{3}^{10}\right)_{0} ;+221079468 \lambda^{5}+49599473 \lambda^{4}$ \\
& & & $-1529140 \lambda^{3}+27675 \lambda^{2}$ \\
& & & $-243 \lambda+1$ \\
\hline 2 & $\left(0,0,-\frac{1}{2}, 1,1, \frac{1}{2}, 0^{*}\right)$ & $\psi^{-1 / 2}$ & $\lambda=\left(b_{3}\right)_{0} ; \lambda^{10}-62208$ \\
\hline 3 & $\left(\frac{1}{7}, 0,-\frac{1}{7}, \frac{2}{7},-\frac{4}{7},-\frac{5}{7}, 0^{*}\right)$ & $\psi^{-5 / 7}$ & $\lambda=\left(2 a_{3}^{2} a_{2}\right)_{0} ; \lambda^{14}-5 \lambda^{7}+5$ \\
\hline 4 & $\left(0, \frac{1}{2}, \frac{1}{2},-\frac{1}{2}, 0^{*}, \frac{1}{2}, \frac{1}{2}\right)$ & $\psi^{-5 / 2}$ & $\lambda=\left(32 b_{3}^{5}\right)_{0} ; \lambda^{4}+11 \lambda^{2}-1$ \\
\hline
\end{tabular}

Remembering the warnings emitted in Section 5.1, we have a little bit of extra work to do at low order: the equations at lowest order might not determine all $\left(v_{i}\right)_{0}$ immediately. They could also split into several pieces that lie on separate components of $\mathcal{H}_{\text {conics. }}$. For the latter issue, we keep only those that lie on $\mathcal{I}_{2}$, i.e., which satisfy (3.31). For the former, we continue to higher order. This determines the local expansion parameter $\psi^{-\beta}$, and eventually, all obstructions are lifted, and we can mechanically solve the recursion. We identify (a power of) one of the $\left(v_{i}\right)_{0}$ as generator of the number field associated with the corresponding group of branches. The information on the various groups belonging to $\mathcal{I}_{2}$ is collected in table 1 .

To illustrate the complexity, we give some of the lowest order terms in the expansion of the fourth group in the table:

$$
\begin{aligned}
a_{1} a_{2}= & \frac{-8 \lambda-\lambda^{3}}{20} \psi^{1 / 2}+\frac{-47-4 \lambda^{2}}{20} \psi^{-2} \\
& +\frac{130229 \lambda+11743 \lambda^{3}}{40} \psi^{-9 / 2}+\ldots, \\
a_{2}^{5}= & \frac{\lambda^{3}}{32} \psi^{5 / 2}+\frac{3+2 \lambda^{2}}{32}+\frac{-1105 \lambda-101 \lambda^{3}}{64} \psi^{-5 / 2}+\ldots,
\end{aligned}
$$




$$
\begin{aligned}
a_{3}^{2} / a_{2}^{4}= & -\frac{16\left(21+2 \lambda^{2}\right)}{15} \psi^{-1}+\frac{32\left(5024 \lambda+453 \lambda^{3}\right)}{15} \psi^{-7 / 2} \\
& -\frac{16\left(71099+6411 \lambda^{2}\right)}{3} \psi^{-6}+\ldots
\end{aligned}
$$

We have also ventured into the calculation of the inhomogeneous PicardFuchs equation for these cycles. Working order by order in the residue calculus of [1], we obtain for the third group in Table 1:

$$
\begin{aligned}
f(z)= & \frac{i \lambda^{1 / 2}}{4 \pi^{2}}\left[\frac{25\left(5 \lambda^{5}-\lambda^{12}\right)}{343} z^{1 / 7}+\frac{500\left(94-17 \lambda^{7}\right)}{2401} z^{2 / 7}\right. \\
& +\frac{225\left(70585 \lambda^{2}-31748 \lambda^{9}\right)}{16807} z^{3 / 7}+\frac{400\left(2394125 \lambda^{4}-191028 \lambda^{11}\right)}{117649} z^{4 / 7} \\
& \left.+\frac{3875\left(245997065 \lambda^{6}-63500311 \lambda^{13}\right)}{823543} z^{5 / 7}+\cdots\right]
\end{aligned}
$$

(as usual, $z=(5 \psi)^{-5}$ ). This illustrates again the general structure we have been discussing: the seventh root of unity is the phase of the local expansion parameter $z^{1 / 7}$. The additional square-root in (5.35) originates from the choice of sign of $a_{3}$ in (5.32): the exchange of $x_{3}$ and $x_{4}$ changes the cycle class by a sign. The remaining irrationality is intrinsic to the group of algebraic cycles under consideration.

\section{Expansion in large volume limit}

We are now ready to study the A-model expansion of the space-time superpotential. The main focus is the so-called multi-cover formula that relates the A-model expansion to the BPS content of the supersymmetric spacetime theory.

Schematically, the general prediction of [5] was that a single BPS state of charge $\beta$ should make a contribution to the space-time superpotential of the form

$$
\mathcal{W}_{\beta}(q) \sim \operatorname{Li}_{2}\left(q^{\beta}\right) \sim \sum_{k} \frac{q^{\beta k}}{k^{2}}
$$

where $t=\log q$ is the complex scalar in the supermultiplet coupling to $\beta$, and $\mathrm{Li}_{2}$ is the standard Euler's di-logarithm function. The sum over $k$ originates as the Laplace transform of the D0-brane charge in the M-theory derivation of (6.1). In the context of [5] one assumes a local A-model setup with a 
non-compact Lagrangian as D-brane, where $t$ represents Kähler moduli as well as freely adjustable D-brane moduli.

If $n_{\beta}$ is the degeneracy of BPS states of charge $\beta$, the total superpotential is

$$
\mathcal{W}=\sum_{\beta} n_{\beta} \mathcal{W}_{\beta}
$$

This superpotential, together with its higher-derivative generalizations in the context of the open topological string, is equivalently computable from a sum over world-sheet instantons with boundary on the background D-brane. Disentangling the contributions in the various charge sectors, see, e.g., [25], leads to the customary relations between open Gromov-Witten invariants and BPS (Ooguri-Vafa) invariants.

For example, for the standard ("inner") brane on the (resolved) conifold at zero framing, there are two BPS states of charge $(0,1)$ and $(1,-1)$, respectively, with a space-time superpotential:

$$
\mathcal{W}(t, u)=\sum_{k=1}^{\infty}\left(\frac{\mathrm{e}^{k u}}{k^{2}}+\frac{\mathrm{e}^{k(t-u)}}{k^{2}}\right),
$$

for the Kähler modulus $t$, and the open string (D-brane) modulus $u$. In the worldsheet computation of (6.3), one counts holomorphic maps from the disk to the conifold, with boundary mapping to the Lagrangian submanifold wrapped by the D-brane. The sum over $k$ originates from those maps that factor via degree $k$ multi-coverings of the disk by itself, such as

$$
z \mapsto z^{k}
$$

It has been noted in $[2,3,14]$ that these multi-cover formulas are not suitable in the context of compact manifolds. The main physical reason is that anomalies prevent a full separation of open and closed string moduli, while open Gromov-Witten invariants are not defined in general. The basic conundrum is already implicit in [5], where the masses of 2-d BPS solitons are determined by the critical values of the superpotential, which however is only generated by integrating out those very solitons. The issue could be resolved if it were possible to make sense of $\mathcal{W}$ off-shell, i.e., away from its critical points, ideally without additional information from the Kähler potential. In non-compact situations, certain natural choices are suggested by the symmetries of the asymptotic geometry $[6,7]$. In compact situations, 
one class of off-shell choices was proposed in $[12,14]$, and a somewhat different one in [13].

Our way to deal with the ambiguities is to consider the critical points of the superpotential in the $u$-direction (cf., (1.1)). For the conifold,

$$
\begin{aligned}
\partial_{u} \mathcal{W}(t, u) & =-\log \left(1-\mathrm{e}^{u}\right)+\log \left(1-\mathrm{e}^{t-u}\right)=0 \\
& \Rightarrow \mathrm{e}^{u}= \pm \mathrm{e}^{t / 2} .
\end{aligned}
$$

Then the difference of critical values is given by

$$
\mathcal{W}\left(t, u_{+}\right)-\mathcal{W}\left(t, u_{-}\right)=4 \sum_{k \text { odd }} \frac{e^{t k / 2}}{k^{2}}
$$

This on-shell superpotential encodes less information than (6.3), but depends on fewer choices. If the inner brane on the conifold as a local model captures enough of the global geometry, one can elevate (6.6) to a multi-cover formula instead of (6.1).

$$
\mathcal{W}(q)=\sum_{d \text { odd }} \tilde{n}_{d} q^{d / 2}=\sum_{d, k \text { odd }} n_{d} \frac{q^{d k / 2}}{k^{2}}
$$

Indeed, this modification of (6.1) was found in [3] to relate the rational open Gromov-Witten invariants $\tilde{n}_{d}$ of the real quintic to integer invariants $n_{d}$, that fit into a larger framework of real enumerative geometry.

In $[2,14]$, other modifications of the di-logarithm were identified, such as

$$
\sum_{3 \nmid k} \frac{q^{d k / 3}}{k^{2}}
$$

albeit without a description of either local or global A-model geometry.

Through the examples of the present paper, we will see that (6.7) and (6.8) are just the simplest versions of a much more elaborate class of "multicover" formulas. The relevance of certain arithmetic functions in these new multi-cover formulas is rather intriguing, and indicative of deeper connections between mirror symmetry and number theory that we hope to explore elsewhere. 


\subsection{Van Geemen lines}

We first return to the van Geemen families of lines. Their inhomogeneous Picard-Fuchs equation was calculated in Section 2,

$$
\mathcal{L} \mathcal{W}_{B}(z)=f_{\text {van Geemen }}(z)
$$

where

$$
\mathcal{L}=\theta^{4}-5 z(5 \theta+1)(5 \theta+2)(5 \theta+3)(5 \theta+4)
$$

$\theta=\frac{d}{d \ln z}, z=(5 \psi)^{-5}$, and, with $1+\omega+\omega^{2}=0$,

$$
f_{\text {van Geemen }}(z)=\frac{1+2 \omega}{4 \pi^{2}} \times \frac{32}{45} \times \frac{\frac{63}{\psi^{5}}+\frac{1824}{\psi^{10}}-\frac{512}{\psi^{15}}}{\left(1-\frac{128}{3 \psi^{5}}\right)^{5 / 2}}
$$

It is straightforward to solve this equation in a power series around $z=0$, and apply the usual mirror map to obtain

$$
\begin{aligned}
\widehat{\mathcal{W}}_{A}(q)= & 4 \pi^{2} \mathcal{W}_{A}(q)=\frac{4 \pi^{2} \mathcal{W}_{B}}{\varpi_{0}}(z(q)) \\
= & \sqrt{-3}\left(140000 q+\frac{11148100000}{3} q^{2}+\frac{5015947794500000}{27} q^{3}\right. \\
& +\frac{330137902935872500000}{27} q^{4}+\frac{76015582693256843498840000}{81} q^{5} \\
& \left.+\frac{57929080529317310275946498060000}{729} q^{6}+\cdots\right) .
\end{aligned}
$$

Instead of being scared away by the growth of the numerators of the expansion coefficients, let us look at the denominators. We define $\tilde{n}_{d}$ as the coefficient of $q^{d}$ :

$$
\widehat{\mathcal{W}}_{A}(q)=\sum_{d=1}^{\infty} \tilde{n}_{d} q^{d}
$$

Remarkably, the $\tilde{n}_{d}$ are not rational numbers, in distinction to all previous examples in the literature. From expansion to large order, we observe that the denominator of $\tilde{n}_{d}$ grows as $3^{d}$, but otherwise contains at most a factor 
of $d^{2}$, i.e., we have

$$
d^{2} 3^{d} \frac{\tilde{n}_{d}}{\sqrt{-3}} \in \mathbb{Z}
$$

Given previous experience, in which the $d^{2} \tilde{n}_{d}$ were always (rational) integers, the result (6.14) could seem a bit disappointing. On the other hand, the denominators are remarkably smaller than those in $\mathcal{W}_{B}$. Roughly speaking, the mirror map reduces $(d !)^{2}$ to $d^{2}$. It is natural to expect that the factors of $d^{2}$ in the denominator can be removed by an appropriate multi-cover formula. It is remarkable that such a formula indeed exists!

On expanding

$$
\widehat{\mathcal{W}}_{A}(q)=\sum_{d=1}^{\infty} n_{d} \sum_{k=1}^{\infty} \frac{\chi(k)}{k^{2}} q^{d k}
$$

where $\chi(k)$ depends on the residue of $k \bmod 3$,

$$
\chi(k)= \begin{cases}0, & k \equiv 0 \bmod 3 \\ 1, & k \equiv 1 \bmod 3 \\ -1, & k \equiv 2 \bmod 3\end{cases}
$$

we find

$$
3^{d} \frac{n_{d}}{\sqrt{-3}} \in \mathbb{Z}
$$

The first few $n_{d}$ are ${ }^{6}$

$$
\begin{aligned}
n_{1} & =\sqrt{-3} 140000 \\
n_{2} & =\sqrt{-3} \frac{11148205000}{3} \\
n_{3} & =\sqrt{-3} \frac{5015947794500000}{27} \\
n_{4} & =\sqrt{-3} \frac{330137902960955725000}{27} \\
\vdots & \vdots
\end{aligned}
$$

\footnotetext{
6 The first convincing case is $\tilde{n}_{11}=\sqrt{-3} \frac{5195025975738748330135719454410630564027766563867792882680000}{3^{10} 11^{2}}, \quad$ compared with $n_{11}=\tilde{n}_{11}+\tilde{n}_{1} / 11^{2}=$ $\sqrt{-3} \frac{42934098973047506860625780614963888958907161684857860740000}{3^{10}}$
} 
The function $\chi(k)$ in (6.16) is, of course, just the quadratic character modulo 3 , which is the non-trivial Dirichlet character of order 3 , one of the standard arithmetic functions of algebraic number theory.

$$
\chi(k)=\left(\frac{k}{3}\right) .
$$

Incidentally, we may now recognize (6.7) and (6.8) as having a rather similar form, with $\chi(k)$ replaced by the trivial (principal) Dirichlet character of order 2 , and 3 , respectively.

\subsection{The D-logarithm}

The results so far motivate us to introduce more general twists of the di-logarithm, which we will call the D-logarithm, of the form

$$
\mathrm{Li}_{2}^{\mathrm{D}}(x)=\sum_{k=1}^{\infty} \frac{a_{k}}{k^{2}} x^{k},
$$

where $\left(a_{k}\right)$ are sequences of numbers that we will specify (see Sections 6.4 and 6.5).

The purpose of the D-logarithm is to serve as a refinement of the multicover formula (6.1) for general D-brane superpotentials. The notation and terminology, however, is suggested by the special case that $a_{k}=\chi(k)$ is a Dirichlet character, and in which we write,

$$
\mathrm{Li}_{2}^{(\chi)}(x)=\sum_{k=1}^{\infty} \frac{\chi(k)}{k^{2}} x^{k} .
$$

When $\chi$ is a trivial Dirichlet character, we recover the formulas of $[2,3]$, while $\chi(k)=\left(\frac{k}{3}\right)$ is relevant for the van Geemen lines.

The original occurrence of twists of this type of course is in Dirichlet L-functions,

$$
L(s ; \chi)=\sum_{n=1}^{\infty} \frac{\chi(n)}{n^{s}} .
$$

In fact, given the relation of special values

$$
L(2 ; \chi)=\operatorname{Li}_{2}^{(\chi)}(1)
$$


one might view the D-logarithm as a natural alternative analytic continuation of those special values. We will see below however that the coefficients $\left(a_{k}\right)$ relevant for the D-logarithm are in general different from those occurring in typical $L$-series.

\subsection{First component, first group}

So let us consider now the A-model expansion of the superpotential for the family of conics that we have been calling $\mathcal{I}_{1}$, and whose inhomogeneous Picard-Fuchs equation is calculated in (5.27).

As discussed in Section 5 , the 10 branches of $\mathcal{I}_{1}$ over the complex structure moduli space fall into two groups in the large volume limit. The first group contains six branches that are in correspondence with the solutions of (5.24); the second has four branches and is governed by (5.26). We do not have an A-model interpretation of this structure, but we can calculate the superpotential as a solution of (5.27). This will allow us to delineate the definition of the D-logarithm.

In the expansion (5.28), we find after the standard mirror map:

$$
\begin{aligned}
\widehat{\mathcal{W}}_{A}(q)= & 600 \cdot 5^{2 / 3} \cdot q^{1 / 6}-100150 q^{1 / 2}+\frac{30155000}{3} \cdot 5^{1 / 3} \cdot q^{5 / 6} \\
& -\frac{1965946252000}{1323} \cdot 5^{2 / 3} \cdot q^{7 / 6}+\frac{12016906931000}{9} q^{3 / 2} \\
& -\frac{7939808112480350000}{29403} \cdot 5^{1 / 3} \cdot q^{11 / 6} \\
& +\frac{21851198476716995185000}{369603} \cdot 5^{2 / 3} \cdot q^{13 / 6} \\
& -\frac{205546823520516323768}{3} q^{5 / 2} \\
& +\frac{94301250909743023365521125000}{5688387} \cdot 5^{1 / 3} \cdot q^{17 / 6} \\
& -\frac{795791304680793507631175999075000}{191850201} \cdot 5^{2 / 3} \cdot q^{19 / 6} \\
& +\frac{783657804098608936611454866250}{147} q^{7 / 2}+\ldots
\end{aligned}
$$

We have here substituted $\lambda=5^{2 / 3}$ in order to facilitate the following observations. Denoting the coefficient of $q^{d / 6}$ by $\tilde{n}_{d}$, we have (all of what follows is confirmed to rather high order, up to $d \gtrsim 600$ )

- when $2 \mid d, \tilde{n}_{d}=0$

- when $3 \mid d, d^{2} \tilde{n}_{d} \in \mathbb{Z}$ 
- when $(d, 6)=1, d^{2} 3^{\left\lfloor^{\frac{3 d}{4}}\right\rfloor} \tilde{n}_{d} \in 5^{\frac{2}{3}} \mathbb{Z}$ when $d=1 \bmod 3$ and $\in 5^{\frac{1}{3}} \mathbb{Z}$ when $d=$ $2 \bmod 3$.

There are some similarities, but also noticeable differences to (6.12):

- The irrationality of the $\tilde{n}_{d}$ is not just an overall factor for $\widehat{\mathcal{W}}_{A}$.

- The denominators of the irrational $\tilde{n}_{d}$ grow with $d$ as a power of 3 , although slightly less rapidly than (6.12).

- The $\tilde{n}_{d}$ for $3 \mid d$ are integer up to a factor of $d^{2}$.

As for the van Geemen lines (6.15), our goal now is to describe the D-logarithm such that via

$$
\widehat{\mathcal{W}}_{A}(q)=\sum_{d} \tilde{n}_{d} q^{d / 6}=\sum_{d} n_{d} \mathrm{Li}_{2}^{\mathrm{D}}\left(q^{d / 6}\right)=\sum_{d} n_{d} \sum_{k} \frac{a_{k}}{k^{2}} q^{d k / 6}
$$

the $n_{d}$ will have the remaining $d^{2}$ dropped from their denominators. Some important points are clear at this stage already.

* The $n_{d}$ will remain irrational, and we are prepared to live with a growing denominator.

* The $a_{k}$ appearing in the D-logarithm will also be irrational. The most natural way to capture the symmetries of (5.24) is that the $a_{k}$ are rational up to a factor of $5^{2 d(k-1) / 3}$.

* This means in particular that the $a_{k}$ must depend on $d$. Given the symmetries, one expects that the $a_{k}$ will depend on $d \bmod 3$.

Before looking for a solution to these constraints, we add the following piece of information: in a putative physics interpretation, to be further discussed below, the $n_{d}$ should be related to a degeneracy of appropriate BPS states that multiplies those states' contribution to the space-time superpotential, see Equation (6.2). Looking back at Equations (6.7), (6.8) and (6.15), we see that the variations of the multi-cover formula are related to the vacuum structure of the associated D-brane configuration, and more precisely to the action of the symmetry group of the algebraic equation determining that structure, the equation being $\varphi^{2}=1, \varphi^{3}=1$ and $\omega^{2}+\omega+1=0$ in those three cases, respectively. Together with the present data, this indicates that the correct version of the Ooguri-Vafa formula (6.1) depends on the arithmetic properties of the "BPS degeneracies" $n_{d}$, and more specifically on the action of the Galois group of the relevant number field.

A first hint that the result (6.24) fits into the general framework comes from the part of the expansion with only rational coefficients. As remarked above, the $\tilde{n}_{3 d}$ are rational, with denominator $(3 d)^{2}$. And with the simple 
twist by the principal character of order 2 ,

$$
\sum_{d \text { odd }} \tilde{n}_{3 d} q^{d / 2}=\sum_{d, k \text { odd }} \frac{n_{3 d}}{k^{2}} q^{d k / 2}
$$

one finds that the $n_{3 d}$ are indeed integer. This is a quite non-trivial check that there is no simple mistake in (5.27).

The irrational part of (6.24) is governed by the number field $K=\mathbb{Q}\left(5^{1 / 3}\right)$, with Galois completion $L=\mathbb{Q}\left(5^{1 / 3}, \sqrt{-3}\right)$. A crucial observation that sets us onto the right track is the following. Whereas $\tilde{n}_{7}$ and $\tilde{n}_{11}$ do not have an obvious congruence with $\tilde{n}_{1}$ (it is difficult to ascertain any statements about $d=5$ or multiples thereof, because 5 divides the discriminant of the number field), one finds that in the combination

$$
\tilde{n}_{13}-\frac{\tilde{n}_{1}}{13^{2}}=5^{2 / 3} \cdot \frac{129297032406609431200}{3^{7}},
$$

the $13^{2}$ in the denominator cancels out. So we learn that $a_{13}=1$. Invoking rudimentary knowledge of elementary algebraic number theory, we understand that indeed 13 is special with respect to our number theoretic situation: it is a prime that splits completely in the field extension $L / \mathbb{Q}$. Actually, already in $K$,

$$
x^{3}-5=(x+2)(x+5)(x+6) \bmod 13 .
$$

That (6.27) is not a coincidence can be confirmed by checking the next primes with the same property: $67,127, \ldots$.

Now let's again pause and compare with the van Geemen lines: when $k=p$ is prime, $a_{p}=\left(\frac{p}{3}\right)=1$ when $p$ is a quadratic residue $\bmod 3$. By reciprocity, this is the case precisely when $p$ splits completely in $\mathbb{Q}(\sqrt{-3})$, i.e., $x^{2}+3=0$ has two solutions in $\mathbb{Z} / p$. This is clearly consistent with the observations around (6.28). When $p$ does not split in $\mathbb{Q}(\sqrt{-3}), a_{p}=-1$. More generally we have the multiplicativity $a_{k_{1} k_{2}}=a_{k_{1}} a_{k_{2}}$.

Adding knowledge of all previous cases as well as (6.26) gives us a very clear understanding of when $a_{p}=1$ : either there is no field extension, or $p$ splits completely in the extension field. But how do we generalize the non-trivial $\left(\frac{p}{3}\right)$ when $K=\mathbb{Q}\left(5^{1 / 3}\right)$, or some other number field? Since $L=K(\sqrt{-3})$ is a non-abelian extension, with Galois group $G=S^{3}$ the full permutation group, consultation of the literature might suggest to 
try characters of higher-dimensional representations of $G$. This however is incompatible with the expectation that the $a_{p}$ should themselves be general irrationals, and seems in fact impossible to realize. Nevertheless, some detective's work reveals that the correct answer indeed involves the structure of $G=\operatorname{Gal}(L / \mathbb{Q})$, especially its action at the primes. We explain this next.

\subsection{D-logarithm $\bmod k^{2}$}

In the previous subsections, we have listed various constraints that we expect the D-logarithm to satisfy and collected hints that its underlying sequence $\left(a_{k}\right)$ is determined by the arithmetic properties of the invariants $n_{d}$. The for us characteristic property, namely that the formula (6.25) clear the $d^{2}$ from the denominators, can be used to determine the $a_{k}$ as algebraic numbers (integers) $\bmod k^{2}$. This is what we do here. ${ }^{7}$

Based on the cases involving Dirichlet characters, we believe that in general there are distinguished representatives for $a_{k}$, which then define the D-logarithm $\operatorname{Li}_{2}^{\mathrm{D}}(x)$ as an analytic function of $x$. These distinguished representatives should be obtained either from a proper physics derivation of (6.1), or an appropriate mathematical interpretation of the D-logarithm as a multi-cover formula.

We first make explicit that the D-logarithm depends on $n_{d}$ by rewriting (6.25) as

$$
\widehat{\mathcal{W}}_{A}(q)=\sum_{d} \tilde{n}_{d} q^{d / 6}=\sum_{d} n_{d} \sum_{k} \frac{a_{k}^{(d)}}{k^{2}} q^{d k / 6}
$$

with the understanding that $a_{k}^{(d)}$ depends on the upper index $d \bmod 3$. We know that $a_{k}^{(0)}=1$ for all odd $k$ and $a_{p}^{(d)}=1$ when $p$ is a prime that splits completely in $K=\mathbb{Q}\left(5^{1 / 3}\right) .^{8}$

Plugging (6.24) into (6.29) we extract the following values for $a_{k}^{(d)} \bmod$ $k^{2}$ for $k=p$ the first few primes, $d=1,2$ : (We are continuing to write $\lambda=$

\footnotetext{
${ }^{7}$ We give here a bottom-up presentation of the relevant results, following more or less the path along which we came to them. A more straight-forward mathematical definition will be written up elsewhere.

${ }^{8}$ We will not make statements for $k$ that are not co-prime with the discriminant of the number field, which for our given $K$ is equal to $-3^{3} \times 5^{2}$.
} 
$5^{2 / 3}$ but of course other cube roots of $5^{2}$ would do as well.)

\begin{tabular}{c|c|c}
\hline$p$ & $a_{p}^{(1)} \bmod p^{2}$ & $a_{p}^{(2)} \bmod p^{2}$ \\
\hline 7 & 30 & 18 \\
11 & $82 \cdot 5^{2 / 3}$ & $103 \cdot 5^{1 / 3}$ \\
13 & 1 & 1 \\
17 & $247 \cdot 5^{2 / 3}$ & $150 \cdot 5^{1 / 3}$ \\
19 & 68 & 292 \\
23 & $59 \cdot 5^{2 / 3}$ & $477 \cdot 5^{1 / 3}$ \\
29 & $538 \cdot 5^{2 / 3}$ & $700 \cdot 5^{1 / 3}$ \\
31 & 521 & 439 \\
$\vdots$ & $\vdots$ & $\vdots$ \\
\hline
\end{tabular}

The structure is fairly obvious: when $p \equiv 1 \bmod 3$ (but does not split completely), $a_{p}^{(1)}$ and $a_{p}^{(2)}$ are the two roots $\bmod p^{2}$ of the equation

$$
a^{2}+a+1=0
$$

When $p \equiv 2 \bmod 3$, we find that $b^{(1)}=a_{p}^{(1)} / 5^{2 / 3}$ is the root $\bmod p^{2}$ of the equation

$$
25 b^{3}=1
$$

while $b^{(2)}=a_{p}^{(2)} / 5^{1 / 3}$ is the root $\bmod p^{2}$ of the equation

$$
5 b^{3}=1 .
$$

The solutions to (6.32) and (6.33) are unique, but how do we discriminate between the two roots of (6.31)? It turns out that the relevant information comes from the Frobenius automorphism of the residue field extension at $p$ : the reduction of $a_{p}^{(d)} \bmod p$ should agree with $\left(5^{2 d / 3}\right)^{p-1}$,

$$
a_{p}^{(d)}=\left(5^{2 d / 3}\right)^{p-1} \bmod p,
$$

which then uniquely selects the solution of (6.31). (For example, $30=2=$ $\left(5^{2 / 3}\right)^{6}=5^{4} \bmod 7$.)

This reduction also holds for $p \equiv 2 \bmod 3$ :

$$
\frac{a_{p}^{(1)}}{5^{2 / 3}}=\frac{\left(5^{2 / 3}\right)^{p-1}}{5^{2 / 3}} \bmod p,
$$

and a similar equation holds for $d=2 \bmod 3$. 
The distinction between $p=1$, or 2 , mod 3 lies in the order of the Frobenius automorphism. It is equal to 3 , or 2, respectively, which corresponds exactly to the two non-trivial cycle classes of the Galois group $G=S^{3}$.

To understand that these findings are in agreement with the conditions we knew for $a_{p}^{(d)}=1$, we recall that when $p$ splits completely, or $n_{d}$ is rational, the Frobenius automorphism acts trivially. This is true in the first situation because the local field extension is trivial, and in the latter can also be viewed as a consequence of Fermat's little theorem, $a^{p}=a \bmod p$ for any $a \in \mathbb{Z}$. This theorem also implies that the Frobenius acts on $n_{d}$ in the same fashion as on $5^{2 d / 3}$. Thus, we may summarize:

For $p$ prime, $a_{p}^{(d)}$ is the lift $\bmod p^{2}$ of the Frobenius automorphism at $p$ acting on $n_{d}$.

What about $a_{k}$ when $k$ is not prime? A basic expectation is a multiplicative structure relating $a_{k_{1} k_{2}}$ to $a_{k_{1}} \cdot a_{k_{2}}$. It is clear however that this has to be refined because we have obtained $a_{p}$ only $\bmod p^{2}$. Moreover, the condition that $a_{k}$ should be rational up to a factor $5^{2 d(k-1) / 3}$ (see page 320 ) is not naively compatible with a multiplicative structure for $a_{k}^{(d)}$.

When $k=p_{1} p_{2}$ is the product of two distinct primes, one could imagine fixing representatives of $a_{p_{1}}$ and $a_{p_{2}}$ and require that $a_{p_{1} p_{2}}$ coincide with $a_{p_{1}} a_{p_{2}} \bmod \left(p_{1} p_{2}\right)^{2}$. But this is not sufficient when $k$ is divisible by a higher prime power $p^{e}, e>1$. To deal with this situation we introduce $e$ as an additional index and let

$$
a_{p, e}^{(d)} \text { be the lift } \bmod p^{2 e} \text { of the Frobenius at } p \text { acting on } n_{d} \text {. }
$$

For instance, when $p=1 \bmod 3, a_{p, e}^{(d)}$ is the unique solution of $(6.31) \bmod p^{2 e}$ that agrees with the Frobenius $\bmod p$. We agree that $a_{p}^{(d)}=a_{p, 1}^{(d)}$.

The issue with the irrationality of the multiplicative structure can be resolved by mixing the two sequences $a_{k}^{(1)}$ and $a_{k}^{(2)}$.

Here then is the explicit algorithm that allows the recursive calculation of all $a_{k}^{(d)} \bmod k^{2}$. For any prime $p$ dividing $k$, we denote by $e_{p}$ the largest power of $p$ dividing $k$, and we require

$$
a_{k}^{(d)}=a_{p, e_{p}}^{(d k / p)} \cdot a_{k / p}^{(d)} \bmod p^{2 e_{p}}
$$


Imposing these conditions for all primes dividing $k$ determines $a_{k}^{(d)}$ uniquely $\bmod k^{2}$.

An interesting aspect of equation (6.38) is that the value of $a_{k}^{(d)}$ depends recursively on the representatives chosen for $a_{k / p}^{(d)} \bmod k^{2}$ (which is previously determined only $\left.\bmod (k / p)^{2}\right)$. The structure of $(6.29)$ however is such that those choices do not affect the integrality properties of the resulting $n_{d}$. This means that the representatives for $a_{k}^{(d)} \bmod k^{2}$ are not independent from one another, and is good evidence that more distinguished representatives should exist.

How this really works in detail is, of course, clearest in the examples. We have seen above that $a_{7}^{(1)}=30 \bmod 7^{2}$, and easily find $a_{7,2}^{(1)}=1353 \bmod 7^{4}$, so that $a_{49}^{(1)}=40590$. Stripping the mods and solving (6.29), we find

$$
\begin{aligned}
n_{1} & =\tilde{n}_{1}=5^{2 / 3} \times 600 \\
n_{7} & =\tilde{n}_{7}-\frac{30}{7^{2}} n_{1}=-5^{2 / 3} \times \frac{40121362000}{3^{3}} \\
n_{49} & =\tilde{n}_{49}-\frac{30}{7^{2}} n_{7}-\frac{40590}{7^{4}} n_{1}=5^{2 / 3} \times \frac{392867 \cdots 993000}{3^{33}}
\end{aligned}
$$

Plugging one into the other, we see that

$$
\begin{aligned}
n_{49} & =\tilde{n}_{49}-\frac{a_{7}^{(1)}}{7^{2}} \tilde{n}_{7}-\left(\frac{a_{49}^{(1)}}{7^{4}}-\frac{\left(a_{7}^{(1)}\right)^{2}}{7^{4}}\right) n_{1} \\
& =\tilde{n}_{49}-\frac{a_{7}^{(1)}}{7^{2}} \tilde{n}_{7}-\frac{\left(a_{7,2}^{(1)}-a_{7}^{(1)}\right) a_{7}^{(1)}}{7^{4}} n_{1} \\
& =\tilde{n}_{49}-\frac{a_{7,2}^{(1)}}{7^{2}} \tilde{n}_{7}+\frac{a_{7,2}^{(1)}-a_{7}^{(1)}}{7^{2}}\left(\tilde{n}_{7}-\frac{a_{7}^{(1)}}{7^{2}} n_{1}\right) .
\end{aligned}
$$

Since $a_{7,2}^{(1)}=a_{7}^{(1)} \bmod 7^{2}$, this shows that we may change $a_{7}^{(1)}$ by multiples of $7^{2}$ without affecting the fact that the denominator of $n_{49}$ is not divisible by 7 .

We have checked up to $d \gtrsim 600$ that using the three formulas (6.36), (6.37) and (6.38) in (6.29) returns $n_{d}$ with no $d^{2}$ in the denominator.

\subsection{First component, second group}

The definition of the D-logarithm $\bmod k^{2}$ that we have given in the previous subsection was slanted towards the example (6.24). To see that the 
prescriptions (6.36), (6.37) and (6.38) make sense, and are correct, in more generality, we here study the second group of branches of the first component $\mathcal{I}_{1}$ of conics on the mirror quintic.

The A-model expansion in this example begins

$$
\begin{aligned}
\widehat{\mathcal{W}}_{A}(q)= & 10000 \lambda\left(-7624+4517 \lambda^{2}\right) q \\
& -\frac{3200000 \lambda\left(-40650831529+24084846092 \lambda^{2}\right)}{51} q^{2} \\
& +\frac{250000 \lambda\left(-5248611469517402890552+3109702672077500263451 \lambda^{2}\right)}{3^{3} \cdot 17^{2}} q^{3} \\
& -\frac{2500000 \lambda\left(-781124731396525415521048504088+462801576865994098449442008739 \lambda^{2}\right)}{3^{3} \cdot 17^{3}} q^{4} \\
& +\ldots
\end{aligned}
$$

where $\lambda$ is a root of

$$
5 \lambda^{4}+20 \lambda^{2}-48=0 .
$$

The Galois completion of $K=\mathbb{Q}(\lambda)$ is $L=K(\sqrt{-3 / 5})$, with Galois group the dihedral group $D_{4}$. This can be visualized by arranging the four roots of (6.42) in a square,

$$
\begin{array}{cc}
\sqrt{-2+2 \sqrt{\frac{17}{5}}} & \sqrt{-2-2 \sqrt{\frac{17}{5}}} \\
-\sqrt{-2-2 \sqrt{\frac{17}{5}}}--\sqrt{-2+2 \sqrt{\frac{17}{5}}} .
\end{array}
$$

The discriminant of $K$ is $-3 \times 5^{3} \times 17^{2}$, and we may check that the denominators in (6.41) behave as $d^{2} 3^{d} 17^{d}$. We want to remove the $d^{2}$ using the appropriate D-logarithm.

The symmetries in this example do not completely constrain the irrationality of the $\tilde{n}_{d}$, which are general linear combinations of $\lambda$ and $\lambda^{3}$. In other words, only the behavior of $\widehat{\mathcal{W}}_{A}$ under $\pi$-rotation of (6.43) is fixed. As a consequence, the D-logarithm has a more severe dependence on $d$ than in the previous example, leading to even more intricate checks of the formalism.

So let us explain how (6.36) is implemented in the present example. We assume that $n_{d}$ is an algebraic number with denominator vanishing at most 
at the discriminant of $K$, and want to determine $a_{p}$ when $p$ does not divide the discriminant.

As one learns in algebraic number theory, the structure of $K / \mathbb{Q}$ at the (rational) prime $p$ is related to the factorization $\bmod p$ of the minimal polynomial of an integral generator of $K$. In our examples, we could choose $\mu=5 \lambda$, with minimal polynomial

$$
P=\mu^{4}+100 \mu^{2}-6000 .
$$

Then to each factor of $P \bmod p$ is associated a prime (ideal) $\mathfrak{p}_{i}$ in $K$ "lying over $p$ ", and the degree of that factor is the degree of the associated residue field extension $\left(\mathbb{Z}_{L} / \mathfrak{p}_{i}\right) /(\mathbb{Z} / p)$. (As above, $L$ is the Galois completion of $K$, and $\mathbb{Z}_{L}$ is the ring of integers in L.) This being an extension of a finite field, it has cyclic Galois group generated by a single element, called the Frobenius element $\sigma_{i}=\sigma\left(\mathfrak{p}_{\mathfrak{i}} / p\right)$, which acts as $y \mapsto y^{p}$ in the residue field. In the Galois extension $L$, the Frobenius elements associated with different factors of $p$ would all be conjugate to each other, so determine a conjugacy class in the Galois group $G=\operatorname{Gal}(L / \mathbb{Q})$. In our case, $K / \mathbb{Q}$ is not Galois, so we work with $\sigma_{i}$ that act as definite elements of $G$ on the roots of (6.44).

Now given $n_{d}$ as a (non-zero) algebraic number in $K$ we consider, for each $\mathfrak{p}_{i}$ dividing $p$, the algebraic number

$$
z_{i}=\frac{\sigma_{i}\left(n_{d}\right)}{n_{d}} .
$$

These $z_{i}$ themselves live in $L$, but not generally in $K$, and moreover, depend on $i$. So what do we mean in (6.36) by " $a_{p}$ is given by the action of Frobenius at $p "$ ? The underlying idea, familiar in algebraic number theory, is to work "locally around $p$ ", i.e., approximate numbers $\bmod p$ (or, more generally, $\bmod p^{e}$ for $e>1$ ). In the local approximation, we can both find representatives for $z_{i}$, and interpolate between the different $z_{i}$, as $\mathfrak{p}_{i}$ varies over $p$. Moreover, given an approximation to order $p$, we can lift it $\bmod p^{2}$, and this is our definition of $a_{p}$. The lifts mod $p^{2 e}$, needed in (6.37), are then obtained in a straightforward continuation of this procedure.

In formulas, $a_{p}$ is the number in $\mathbb{Z}_{K} / p^{2}$ that agrees with $z_{i}$ at each prime $\mathfrak{p}_{i}$ dividing $p$,

$$
a_{p}=z_{i} \bmod \mathfrak{p}_{i}^{2} .
$$


As in the previous examples, we have found this procedure (augmented with (6.37) and (6.38) for $k$ not prime) such that via

$$
\widehat{\mathcal{W}}_{A}=\sum_{d} \tilde{n}_{d} q^{d}=\sum_{d} n_{d} \sum_{k} \frac{a_{k}}{k^{2}} q^{d k},
$$

it returns invariants $n_{d}$ with no $d^{2}$ in the denominator, up to some significant order $d$.

To make the procedure easier to follow, we discuss as an example, the results for

$$
n_{1}=10000 \lambda\left(-7624+4517 \lambda^{2}\right)
$$

(For incidental reasons, we revert here to the non-integral generator $\lambda$. This does not change the results.) Clearly, under $\lambda \mapsto-\lambda, n_{1} \mapsto-n_{1}$, so $z=-1$. For any element $g \in G$ of the Galois group that reverses the sign under the square-roots in (6.43) (namely, rotation by $\pi / 2$ (order 4 ) or horizontal or vertical flip (order 2$)$ ), the resulting $z=g\left(n_{1}\right) / n_{1}$ is a root of the polynomial

$$
583443+135146154523047386 z^{2}+583443 z^{4} .
$$

Because $583443=3^{5} \times 7^{4}$, but 7 does not divide the discriminant of $K$, that prime will require a bit of a special treatment.

For the first few non-trivial primes, we find the following table

\begin{tabular}{c|c|c|c}
$(6.50)$ & \\
\hline$p$ & $P / 5^{3} \bmod p$ & $f_{p}:=\left(n_{1}\right)^{p-1} \bmod p$ & $a_{p} \bmod p^{2}$ \\
\hline 7 & $(2+\lambda)(5+\lambda)\left(1+\lambda^{2}\right)$ & $2+3 \lambda^{2}$ & $40+20 \lambda^{2}$ \\
11 & $8+4 \lambda^{2}+\lambda^{4}$ & $2+7 \lambda^{2}$ & $24+62 \lambda^{2}$ \\
13 & $6+4 \lambda^{2}+\lambda^{4}$ & $4+12 \lambda^{2}$ & $43+25 \lambda^{2}$ \\
17 & $(7+\lambda)^{2}(10+\lambda)^{2}$ & ramifies & -1 \\
19 & $\left(7+\lambda^{2}\right)\left(16+\lambda^{2}\right)$ & 18 & 1 \\
23 & $(1+\lambda)(8+\lambda)(15+\lambda)(22+\lambda)$ & 1 & $48+417 \lambda^{2}$ \\
29 & $2+4 \lambda^{2}+\lambda^{4}$ & $19+11 \lambda^{2}$ & $235+445 \lambda^{2}$ \\
31 & $\left(3+8 \lambda+\lambda^{2}\right)\left(3+23 \lambda+\lambda^{2}\right)$ & $18+11 \lambda^{2}$ & $780+390 \lambda^{2}$ \\
37 & $(14+\lambda)(23+\lambda)\left(15+\lambda^{2}\right)$ & $3+20 \lambda^{2}$ & $\vdots$ \\
$\vdots$ & $\vdots$ & $\vdots$ &
\end{tabular}

How did we find the last column? From the degree of the factors of $P \bmod p$, we may read off the order of the various Frobenius elements. In most cases, this determines them completely: for inert primes such as 11, 13 and 29, 
the Frobenius has order 4 , so must be rotation by $\pi / 2$ in (6.43). For primes that split completely such as $p=23$, the Frobenius is trivial (as we were happy to learn some time ago!). For primes with one quadratic, and two linear factors, such as 7,37, the Frobenius must be a diagonal flip. The only ambiguous cases are those with two quadratic factors, which could correspond to horizontal/vertical flip, or rotation by $\pi$.

It is easy to check that for $p=11,13,29$ and $31, f_{p}$ solves $(6.49) \bmod p$, and $a_{p}$ is simply the lift of that solution $\bmod p^{2}$. In particular, the Frobenius at 31 must be horizontal/vertical flip.

For $p=19, f_{19}=-1$ at both factors, so Frobenius must be rotation by $\pi$. We keep $a_{19}=-1 \bmod 19^{2}$, just as we use $a_{23}=1$ since 23 splits completely.

For $p=37, f_{37}=1$ at the linear factors, and $f_{37}=-1$ at the quadratic factor. In other words, $f_{37}$ is a solution of $z^{2}-1=0 \bmod 37$, and $a_{37}$ is the lift of that solution mod $37^{2}$.

What happened at $p=7$ ? From the structure of the factorization, it should be in the same class as $p=37$. However, $\left(f_{7}\right)^{2}-1 \neq 0 \bmod 7 \ldots \ldots$ Some reflection reveals that the denominator of $z$ in (6.49) being divisible by 7 is due to the fact that $n_{1}$ vanishes at the two linear factors of 7 , so the action of the Frobenius automorphism as $\left(n_{1}\right)^{p-1}$ becomes completely ambiguous there. Independently however, we have known that the Frobenius should restrict to 1 at the linear factors, and to -1 at the quadratic factor. This can be used to determine that $a_{p}=5+6 \lambda^{2} \bmod 7$, which as a solution of $z^{2}-1=0$ may then safely be lifted mod $7^{2}$.

We may summarize the A-model discussion by stating that once again the Ooguri-Vafa multi-cover formula (6.1) has proven to be basically correct, but that it needs a significant refinement in arithmetically non-trivial situations, which we have encountered here for the first time. The refinement is provided by the D-logarithm, which we conjecture is an analytic function attached to individual algebraic numbers $n_{d}$. The sequences $\left(a_{k}\right)$ defining the D-logarithm are specified $\bmod k^{2}$ by studying the action of the Galois group on $n_{d}$. It remains to be seen whether this remarkable structure can be sharpened and explained more fundamentally, and how it ties in with the rest of our subject. In the remaining Section 7 , we will present some initial thoughts that make this not impossible.

\subsection{Main component}

The only purpose of this subsection is to point out that it is possible to calculate the large volume expansion of the superpotential also on the main component of $\mathcal{H}_{\text {conics }}$, which we called $\mathcal{I}_{2}$ in Section 5. Consider the third 
group in table 1 . We solve the Picard-Fuchs equation with inhomogeneity (5.35), apply the mirror map, and obtain

$$
\begin{aligned}
\frac{4 \pi^{2}}{(-\lambda)^{1 / 2}} \mathcal{W}_{A}= & -175 \lambda^{5}\left(-5+\lambda^{7}\right) q^{1 / 7}-\frac{125\left(-94+17 \lambda^{7}\right)}{4} q^{2 / 7} \\
& -\frac{25 \lambda^{2}\left(-70585+31748 \lambda^{7}\right)}{63} q^{3 / 7}-\frac{25 \lambda^{4}\left(-2394125+191028 \lambda^{7}\right)}{2^{4} \cdot 7^{2}} q^{4 / 7} \\
& -\frac{31 \lambda^{6}\left(-245997065+63500311 \lambda^{7}\right.}{5 \cdot 7^{3}} q^{5 / 7} \\
& -\frac{5 \lambda\left(-8907388019619+2655707519021 \lambda^{7}\right)}{2^{2} \cdot 3^{3} \cdot 7^{4}} q^{6 / 7} \\
& -\frac{5 \lambda^{3}\left(-3595649177+980861072 \lambda^{7}\right)}{7} q \\
& -\frac{\lambda^{5}\left(-1905271484195274460+512248788482392343 \lambda^{7}\right)}{2^{7} \cdot 7^{7}} q^{8 / 7}+\ldots
\end{aligned}
$$

where $\lambda$ is the algebraic number with minimal polynomial

$$
\lambda^{14}-5 \lambda^{7}+5=0 .
$$

It is difficult to obtain convincing tests of our general formalism from (6.51), but the first few orders in the expansion are encouraging: $p=2,5,7$ divide the discriminant, so are allowed in the denominator. Subtraction of $\tilde{n}_{1}$ from $\tilde{n}_{3}$ works as expected, with $a_{3}^{(1)}=8 \lambda^{4}+2 \lambda^{11}$. I have no explanation for the apparent anomaly at $d=6$ (which has $3^{3}$ in the denominator).

\section{Discussion}

In this work, we have studied families of algebraic cycles on the mirror quintic represented by curves of low degree. We have obtained a fairly complete picture of those conics that deform with the mirror quintic. We have seen how the Newton-Puiseux expansion around large complex structure limit splits the algebraic cycle into groups, each governed by an algebraic number field. We have then calculated the truncated normal function (up to an additive constant) by solving the inhomogeneous Picard-Fuchs equation. The irrationality does not disappear after application of the mirror map, confirming a long-standing expectation. To exhibit the underlying (algebraic) integrality ${ }^{9}$ of the expansion, we have introduced the D-logarithm as an arithmetic twist of the di-logarithm. This formalism generalizes all previously known

\footnotetext{
${ }^{9}$ One should work with a notion of integrality that requires a non-negative valuation at all primes except those that ramify.
} 
cases and we might expect that it is complete. Indeed, we formulate the main computational result of this paper as follows:

Conjecture: The A-model (q-)expansion of the truncated normal function associated with an algebraic cycle takes the form

$$
\widehat{\mathcal{W}}_{A}(q)=\sum_{d} \tilde{n}_{d} q^{d / r}
$$

where $r \in \mathbb{Z}_{>0}$, and the $\tilde{n}_{d}$ live in an algebraic number field $K$, with $d^{2} \tilde{n}_{d}$ singular at most at the discriminant of $K$.

In the expansion

$$
\widehat{\mathcal{W}}_{A}(q)=\sum_{d} n_{d} \operatorname{Li}_{2}^{\mathrm{D}}\left(q^{d / r}\right),
$$

the $n_{d}$ themselves are singular at most at the discriminant of $K$. Here, the D-logarithm

$$
\operatorname{Li}_{2}^{\mathrm{D}}(x)=\sum_{k=1}^{\infty} \frac{a_{k}}{k^{2}} x^{k}
$$

is an analytic function that may be attached to any such algebraic number $n_{d}$. The coefficients $a_{k}$ are determined $\bmod k^{2}$ by studying the action of the Galois group on $n_{d}$.

(We did not state all assumptions explicitly, such as that we are on a family of Calabi-Yau three-folds, expand around a large complex structure limit, etc. The allowed singularities include denominators whose order at the discriminant grows (say linearly) with $d$. It should also be clear that we expect the same to work for higher-dimensional moduli spaces. The statements defining the D-logarithm are (6.36), (6.37) and (6.38).)

It is possible that several ingredients for a proof of the above statements are contained in the work of Vologodsky, Schwarz and Kontsevich, see [26].

Turning to possible interpretations of the result, we recall that the truncated normal function gives the contribution to the space-time superpotential of a D-brane configuration that specifies the algebraic cycle, in the B-model. By mirror symmetry, there should be an A-model setup that calculates the expansion (7.1) directly. In our examples, such a setup would contain a Lagrangian submanifold $L \subset X$ of the quintic three-fold, and the $q$-expansion should be the expansion in worldsheet instantons of disc topology.

We lack the tools to exhibit such A-branes directly, but we can nevertheless try to understand whether there is room for the various ingredients: a number field $K$ governing the vacuum structure of $L$, an action of the Galois 
group $G$, and instanton contributions that evaluate to algebraic numbers $\tilde{n}_{d} \in K$.

(i) The irrationality of the instanton contribution is surprising insofar as it has not been seen in any previous example. If anything, $\tilde{n}_{d}$ should be open Gromov-Witten invariants counting holomorphic maps $(D, \partial D) \rightarrow(X, L)$. In all cases studied so far, such invariants always evaluated to rational numbers. Mathematically, the counts are given by intersection theory on moduli spaces $\overline{\mathcal{M}}$ of stable maps as integrals against the virtual fundamental class,

$$
\tilde{n}_{d} \stackrel{?}{\sim} \#\{u:(D, \partial D) \rightarrow(X, L)\} \stackrel{?}{\sim} \int_{[\overline{\mathcal{M}}]^{\mathrm{virt}}} \mathbf{1} .
$$

In general however, open Gromov-Witten invariants have not actually been defined, let alone does there exist a formula like (7.4). The two exceptions are toric manifolds [27] and anti-holomorphic involutions [28]. The main obstacle to doing this in general has long been recognized to be the presence in $\overline{\mathcal{M}}$ of boundaries in real co-dimension one. It is not clear therefore whether there actually exists a good invariant intersection theory on these spaces.

(ii) From the world-sheet point of view, formulas such as (7.4) arise as the reduction of the path-integral to the finite-dimensional space of zeromodes: roughly speaking, because of the vanishing of the fermion kinetic term in the action, one has to pull down the four fermion interaction involving the curvature. In many, favorable, situations, the resulting bosonic integrals have a cohomological interpretation in terms of intersection theory. However, except perhaps with large amounts of supersymmetry, there is no a priori reason why this should happen. It is quite conceivable that in the presence of boundaries, we do not have a strict intersection theoretic interpretation, but the integral still makes sense, and calculates a kind of "volume" of the moduli space. Such a volume could very well evaluate to an algebraic number.

(iii) For a related thought, we recall that ordinary Gromov-Witten invariants are in general not integer, but rational, numbers because of the presence of certain kinds of orbifold singularities in the moduli space. The denominators are the orders of the corresponding identification groups. In the context of open Gromov-Witten theory, the moduli spaces could have other kinds of singularities, such as boundaries and corners, and in particular the latter could potentially make arbitrary irrational contributions.

(iv) Of course, those two options, (ii) and (iii), assume that open Gromov-Witten theory exists in general, and defines actual invariants with an "enumerative" meaning. An alternative attitude is that any such definition 
will depend on arbitrary choices (a common examples being the framing ambiguity of $[7,27])$. With a superpotential interpretation for the open Gromov-Witten invariants, this would mirror the issue, discussed in the introduction, that only the on-shell values of the space-time superpotential have an invariant meaning independent for example of field redefinitions. In this interpretation, the invariants $\tilde{n}_{d}$ would be irrational because they are on-shell and invariant, whereas the actual (rational) counts of discs would happen off-shell, and not be invariant.

(v) This way of looking at the situation is perhaps best suited for explaining how the field extension could arise in the A-model. At the beginning, the underlying Lagrangian $L$ might have non-trivial topology and deformations, which get lifted by those very worldsheet instantons that we are trying to count. Intuitively, the critical points of the superpotential correspond to points in the moduli space of $L$ at which the worldsheet instantons are "balanced" against each other. If there are sufficiently many discs of comparable area, then these critical point conditions will select some general irrational points in the classical moduli space of $L$. If finitely many discs are relevant for this problem, and off-shell counts are rational, then we should be dealing with a finite algebraic extension of the rationals. (At the moment, I do not see how to get infinite, or transcendental extensions in the B-model.)

(vi) Ideally, one would like to understand this in a suitable local model. For customary toric Lagrangian branes however, there are at most two discs determining the critical points, as for the conifold (6.3). A local model realizing a non-trivial field extension is therefore not likely to be toric (and in a sense would not be fully local since disc instantons ending in different places on $L$ would be relevant).

(vii) A local model would also be desirable in order to understand the structure of the multi-cover formula (7.2). Otherwise, we have comparably little to offer for interpreting the invariants $n_{d}$. From the previous discussion in [5], we expect a relation to the spectrum of appropriate BPS states (solitons) interpolating between the supersymmetric vacua. Given that the latter are in correspondence with roots of a polynomial equation, the Galois group of the relevant number field will act also on those BPS states. An irrational "dimension" could be part of the package of these Galois representations. The $a_{k}$ would then be other traces, and the formula (7.2) could perhaps be understood by revisiting the derivation in [5] in light of such results.

For a very brief sampling of other recent works on various ways to relate geometry and physics of Calabi-Yau three-folds with number theory, see $[4,29-33]$ 


\section{Acknowledgments}

I am grateful to Henri Darmon, Hans Jockers, Sheldon Katz, Matt Kerr, Josh Lapan, Wolfgang Lerche, Greg Moore, David Morrison and Noriko Yui for valuable discussions, comments and encouragement. I thank Anca Mustaţă for sharing the results of [24]. I would like to thank the KITP in Santa Barbara for sunny hospitality during the tedious writing of Section 3. Some of the results presented here were also announced at the BIRS Workshop on "Number theory and physics at the crossroads", May 8-13, 2011. Special thanks to Henri Darmon for help in unraveling the D-logarithm. This research is supported in part by an NSERC discovery grant and a Tier II Canada Research Chair. This research was supported in part by DARPA under Grant No. HR0011-09-1-0015 and by the National Science Foundation under grant no. PHY05-51164.

\section{References}

[1] D. R. Morrison and J. Walcher, D-branes and normal functions, Adv. Theor. Math. Phys. 13 (2009), 553, arXiv:hep-th/0709.4028.

[2] J. Walcher, Calculations for mirror symmetry with D-branes, J. High Energy Phys. 09 (2009), 129, arXiv:hep-th/0904.4905.

[3] J. Walcher, Opening mirror symmetry on the quintic, Comm. Math. Phys. 276 (2007), 671-689, arXiv: hep-th/0605162.

[4] G. W. Moore, Arithmetic and attractors, arXiv:hep-th/9807087; Attractors and arithmetic, arXiv:hep-th/9807056.

[5] H. Ooguri and C. Vafa, Knot invariants and topological strings, Nucl. Phys. B 577 (2000), 419, arXiv:hep-th/9912123.

[6] M. Aganagic and C. Vafa, Mirror symmetry, D-branes and counting holomorphic discs, arXiv:hep-th/0012041.

[7] M. Aganagic, A. Klemm and C. Vafa, Disk instantons, mirror symmetry and the duality web, Z. Naturforsch. A 57 (2002), 1, arXiv:hep-th/0105045.

[8] P. Mayr, $N=1$ mirror symmetry and open/closed string duality, Adv. Theor. Math. Phys. 5 (2002), 213, arXiv:hep-th/0108229. 
[9] W. Lerche, P. Mayr and N. Warner, Holomorphic $N=1$ special geometry of open-closed type II strings, arXiv:hep-th/0207259; $N=1$ special geometry, mixed Hodge variations and toric geometry, arXiv:hep-th/0208039.

[10] D. Krefl and J. Walcher, Real mirror symmetry for oneparameter hypersurfaces, J. High Energy Phys. 09 (2008), 031, arXiv:hep-th/0805.0792.

[11] J. Knapp and E. Scheidegger, Towards open string mirror symmetry for one-parameter Calabi-Yau hypersurfaces, arXiv:hep-th/0805.1013; -, Matrix factorizations, massey products and f-terms for twoparameter Calabi-Yau hypersurfaces, arXiv:hep-th/0812.2429.

[12] H. Jockers and M. Soroush, Effective superpotentials for compact D5brane Calabi-Yau geometries, Comm. Math. Phys. 290 (2009), 249, arXiv:hep-th/0808.0761; -, Relative periods and open-string integer invariants for a compact Calabi-Yau hypersurface, Nucl. Phys. B 821 (2009), 535, arXiv:hep-th/0904.4674.

[13] T. W. Grimm, T. W. Ha, A. Klemm and D. Klevers, The D5-brane effective action and superpotential in $N=1$ compactifications, Nucl. Phys. B 816 (2009), 139, arXiv:hep-th/0811.2996; -, Computing brane and flux superpotentials in F-theory compactifications, J. High Energy Phys. 04 (2010), 015, arXiv: hep-th/0909.2025. —, Five-brane superpotentials, blow-up geometries and SU(3) structure manifolds, J. High Energy Phys. 05 (2011), 113, arXiv: hep-th/1011.6375.

[14] M. Alim, M. Hecht, P. Mayr and A. Mertens, Mirror symmetry for Toric branes on compact hypersurfaces, J. High Energy Phys. 09 (2009) 126, arXiv:hep-th/0901.2937; —, H. Jockers and M. Soroush, Hints for off-shell mirror symmetry in type II/F-theory compactifications, Nucl. Phys. B 841 (2010), 303, arXiv:hep-th/0909.1842.

[15] M. Aganagic and C. Beem, The geometry of D-brane superpotentials, J. High Energy Phys. 12 (2011) 060, arXiv:hep-th/0909.2245.

[16] H. Fuji, S. Nakayama, M. Shimizu and H. Suzuki, A note on computations of D-brane superpotential, J. Phys. A 44 (2011), 465401, arXiv:hep-th/1011.2347.

[17] M. Shimizu and H. Suzuki, Open mirror symmetry for Pfaffian Calabi-Yau 3-folds, J. High Energy Phys. 03 (2011), 083, arXiv:hep-th/1011.2350. 
[18] M. Alim, M. Hecht, H. Jockers, P. Mayr, A. Mertens and M. Soroush, Flat connections in open string mirror symmetry, arXiv:hep-th/1110.6522.

[19] A. Neitzke and J. Walcher, Background independence and the open topological string wavefunction, in 'From Hodge theory to integrability and TQFT', ed. R. Donagi and K. Wendland, American Mathematical Society, 2008, arXiv:hep-th/0709.2390.

[20] A. Mustaţă, Degree 1 curves in the Dwork pencil and the mirror family, Math. Ann. 2010, arXiv:math/0311252.

[21] A. Albano and S. Katz, Lines on the fermat quintic threefold and the infinitesimal generalized hodge conjecture, Trans. AMS, 324(0.1) (1991), 353; van Geemen's Families of Lines on Special Quintic Threefolds, Manuscripta Math. 70(2) (1991), 183.

[22] E. Witten, Branes and the dynamics of QCD, Nucl. Phys. B 507 (1997), 658, arXiv: hep-th/9706109.

[23] S. Katz, On the finiteness of rational curves on quintic threefolds, Compos. Math. 60 (1986), 151-162.

[24] A. Mustaţă, A note on higher degree curves in the Dwork pencil of quintics, unpublished, and private communication. See also S. Xu, Degree 2 curves in the Dwork pencil, PhD Dissertation, Ohio State University (2008).

[25] J. M. F. Labastida, M. Marino and C. Vafa, Knots, links and branes at large N, J. High Energy Phys. 11 (2000), 007, arXiv:hep-th/0010102.

[26] A. Schwarz and V. Vologodsky, Integrality theorems in the theory of topological strings, Nucl. Phys. B $\mathbf{8 2 1}$ (2009), 506, arXiv:hep-th/0807.1714.

[27] S. H. Katz and C. C. Liu, Adv. Theor. Math. Phys. 5 (2002), 1, arXiv:math/0103074.

[28] J. Solomon, Intersection theory on the moduli space of holomorphic curves with Lagrangian boundary conditions, MIT thesis, 2006, arXiv: math/0606429.

[29] P. Candelas, X. de la Ossa and F. Rodriguez-Villegas, Calabi-Yau manifolds over finite fields, I, II, Fields Inst. Commun. 38, 121-157, arXiv:hep-th/0012233; arXiv:hep-th/0402133. 
[30] K. Samol and D. van Straten, Frobenius polynomials for Calabi-Yau equations, arXiv:math.AG/0802.3994.

[31] F. Gouveau and N. Yui, Rigid Calabi-Yau threefolds over $\mathbb{Q}$ are modular, Expo. Math. 29 (2011), 142-149, arXiv:math.NT/0902.1466.

[32] G. Bini and B. van Geemen, Geometry and arithmetic of Maschke's Calabi-Yau threefold arXiv:math.AG/1110.01016.

[33] Y.-H. He and J. McKay, $N=2$ Gauge theories: congruence subgroups, coset graphs and modular surfaces, arXiv:hep-th/1201.3633.

Departments of Mathematics and Statistics, and Physics

MCGILl UNIVERSITY

805 Sherbrooke Street W

Montreal, Quebec H3A 0B9

CANADA

RECEIVED FEBRUARY 10, 2012 
